Research Article

\title{
The E-Bayesian Estimation for Lomax Distribution Based on Generalized Type-I Hybrid Censoring Scheme
}

\author{
Kaiwei Liu (iD) and Yuxuan Zhang \\ Department of Mathematics, Beijing Jiaotong University, Beijing 100044, China \\ Correspondence should be addressed to Kaiwei Liu; 18271071@bjtu.edu.cn
}

Received 25 January 2021; Revised 11 April 2021; Accepted 30 April 2021; Published 19 May 2021

Academic Editor: Tushar Jain

Copyright (C) 2021 Kaiwei Liu and Yuxuan Zhang. This is an open access article distributed under the Creative Commons Attribution License, which permits unrestricted use, distribution, and reproduction in any medium, provided the original work is properly cited.

\begin{abstract}
This article studies the E-Bayesian estimation of the unknown parameter of Lomax distribution based on generalized Type-I hybrid censoring. Under square error loss and LINEX loss functions, we get the E-Bayesian estimation and compare its effectiveness with Bayesian estimation. To measure the error of E-Bayesian estimation, the expectation of mean square error (EMSE) is introduced. With Markov chain Monte Carlo technology, E-Bayesian estimations are computed. Metropolis-Hastings algorithm is applied within the process. Similarly, the credible interval for the parameter is calculated. Then, we can compare the MSE and E-MSE to evaluate whose result is more effective. For the purpose of illustration in real datasets, cases of generalized Type-I hybrid censored samples are presented. In order to judge whether the sample data can be directly fitted by the Lomax distribution, we adopt the Kolmogorov-Smirnov tests for evaluation. Finally, we can get the conclusion after comparing the results of E-Bayesian and Bayesian estimation.
\end{abstract}

\section{Introduction}

1.1. Hybrid Censoring Scheme (HCS). Hybrid censoring is a combination of Type-II and Type-I HCS. On HCS definition, when the number of failure samples reaches $k$ or the experimental time reaches $T$, we end the experiment and start to analyze the data, in which $k$ is the number of failed samples in the total $n$ samples and $T$ is a predetermined time.

In Type-I HCS, $T_{*}=\min \left\{T, X_{k: n}\right\}$ is a random end time of the life test experiment. $T$ represents a reasonable time that has been specified, and $X_{k: n}$ represents the time when the $k$-th item fails in the samples. It is fixed in advance that $k \in\{1, \ldots, n\}$. For the experimental time $T$, we define that $T \in(0,+\infty)$. As soon as time $X_{k: n}$ or $T$ is reached, we terminate the life-testing experiment. In Type-II HCS, $T^{*}=$ $\max \left\{T, X_{k: n}\right\}$ is also a random time when the later of $T$ and $X_{k: n}$ is reached. This means that in the test experiment, there must be at least $k$ items that have failed and all of them are sure to be observed.
Both types of HCS have been used under different conditions. The generalized Type-I HCS has been used to study a variety of distribution functions. For example, Rabie and $\mathrm{Li}$ [1] presented the concept of hybrid censoring and studied the E-Bayesian estimation for Burr-X distribution.

Type-II HCS is also widely used. Based on Type-II HCS, Jaheen and Okasha [2] gave the study of the E-Bayesian estimation for Burr Type XII. Based on adaptive Type-II progressive hybrid censoring, Liu and Gui [3] dealt with the estimation of the parameters for Rayleigh distribution.

Though all these studies include various parameter estimations of corresponding functions, the related research for E-Bayesian estimation is rare. There are only some papers that can be referred to, such as [4], [5], and [6].

Either Type-I or Type-II HCS has its own advantages and disadvantages. For example, Type-I HCS has a predetermined time for the experiment. So, we have a per-fixed time to make sure that our experiment will not last too long. But it has the possibility of too few failed items that can be studied before we terminate the experiment. This 
disadvantage, no doubt, affects the efficiency of the estimations and even decreases the accuracy of the results.

On the contrary, Type-II HCS can overcome the disadvantage of Type-I HCS and guarantee enough failed items are obtained; it also has its own drawback. Because it is possible to take more time than expected to get enough failed items, we are not sure how long we need to get the required specified number of failed items.

To solve the above problems, two generalized HCSs are proposed as follows:

For generalized Type-I HCS, we are able to introduce a new integer $r$. The value range of $r$ is the same as that of $k$, which satisfies the requirement $r, k \in\{1,2, \ldots, n\}$. We define $k<r<n$ as the corresponding relation of the three integers. At the same time, we set the experimental time to meet the conditions $T \in(0,+\infty)$. If the $k$-th item fails before the specified time $T$, the end time of the test is set as $\min \left\{T, X_{r: n}\right\}$. On the contrary, if the $k$-th item fails after the specified time $T$, we let $X_{k: n}$ to be the ending time.

For generalized Type-II HCS, we also use the integer $r$. The value range of $r$ is that $r \in\{1,2, \ldots, n\}$. Then, we introduce two new time points, $T_{2}$ and $T_{1}$. Both of them need to obey condition $T_{2}, T_{1} \in(0,+\infty)$ at the same time. We delimit that $T_{2}>T_{1}$. If the failure of the $r$-th item happens before time $T_{1}$, we let $T_{1}$ to be the ending time. If the $r$-th item fails between $T_{1}$ and $T_{2}$, the time for stopping the test is set as $X_{r: n}$. If the $r$-th item fails after time $T_{2}$, we let $T_{2}$ to be the time that the test coming to an end.

We can go over Chandrasekar et al. [7] for more details. Based on Type-I HCS, three types of censored data under different conditions are presented as follows:

(i) Case I: if $X_{k: n}>T$, select $\left\{X_{1: n}<\cdots<X_{k: n}\right\}$;

(ii) Case II: if $X_{r: n}<T$, select $\left\{X_{1: n}<\cdots<X_{r: n}\right\}$;

(iii) Case III: if $X_{r: n}>T$, select $\left\{X_{1: n}<\cdots<X_{d: n}\right\}$,

in which the experimenter chooses a prefixed maximum time $T$ according to how long he can spend for life-testing, $r$ stands for the number of desired failed items, and $k$ stands for the minimum number of failed items acceptable. This type of censored data can improve the effectiveness of the experiment.

1.2. Lomax Distribution. Lomax distribution is a widely used life distribution in reliability and life test research, especially in analyzing the data of life-testing experiments in physics, medicine, biological sciences, and engineering sciences. This distribution has monotonically increasing and decreasing failure rates.

We define the unknown shape parameter as $\alpha>0$ and the known scale parameter as $\lambda>0$. For the Lomax distribution, the probability density function (PDF) is

$$
f(x)=\frac{\alpha}{\lambda}\left(1+\frac{x}{\lambda}\right)^{-(\alpha+1)}, \quad x>0 .
$$

Hence, the cumulative distribution function (CDF) is

$$
F(x)=1-\left(1+\frac{x}{\lambda}\right)^{-\alpha}, \quad x>0, \alpha, \lambda>0 .
$$

$R(t)$, the reliability function, for Lomax distribution is

$$
R(t)=\left(1+\frac{t}{\lambda}\right)^{-\alpha}, \quad \alpha, \lambda>0, t>0 .
$$

$h(t)$, the hazard rate function, is given as

$$
h(t)=\frac{\alpha}{\lambda}\left(1+\frac{t}{\lambda}\right)^{-1}, \quad \alpha, \lambda>0, t>0 .
$$

We need to specify $\alpha>1$ to make the expectation meaningful. When $X \sim \operatorname{Lomax}(\alpha, \lambda)$, the expectation of $X$ is

$$
E(X)=\frac{\lambda}{\alpha-1}, \quad \alpha>1 .
$$

Similarly, we need to specify $\alpha>2$ to make the variance meaningful. The variance of $X$ is

$$
\operatorname{Var}(X)=\frac{\alpha \lambda^{2}}{(\alpha-2)(\alpha-1)^{2}}, \quad \alpha>2 .
$$

The line charts of the PDF of Lomax distribution with different $\alpha$ and $\lambda$ are shown in Figures 1 and 2. The line charts of the hazard function of Lomax distribution with different $\alpha$ and $\lambda$ are shown in Figures 3 and 4 .

When it comes to Figures 1 and 2, the plots of the probability density functions, we can observe the characteristics of the line charts under the different values of $\alpha$ and $\lambda$. We get the charts when $\alpha=8,10,12$ as $\lambda=1$ and when $\lambda=0.8,1,1.5$ as $\alpha=10$. The probability density is concentrated in places where the value of time is smaller as the parameter $\alpha$ increases. As the parameter $\lambda$ increases, probability density decreases in places where the value of time is smaller.

For Figures 3 and 4, the plots of the hazard functions, we can observe the characteristics of the line charts under the different values of $\alpha$ and $\lambda$. We get the charts when $\alpha=$ $8,10,12$ as $\lambda=1$ and when $\lambda=0.8,1,1.5$ as $\alpha=10$. It is obvious that when the values of $\lambda$ and $t$ are constant, the value of $h(t, \alpha)$ increases with the increases of $\alpha$. When the values of $\alpha$ and $t$ are constant, the value of $h(t, \lambda)$ decreases with the increases of $\lambda$. With the increase of $t$, the difference of the values between hazard functions decreases.

The parameters of the Lomax distribution can be estimated in different ways under different conditions. In the study by Al-Sobhi and Al-Zahrani [8], the estimation of the parameters of Lomax distribution was given under general progressive censoring. Labban [9] discussed the two-parameter estimation of Lomax distribution.

At the same time, composite distribution functions of Lomax distribution have been studied too. Alzahrani and Sagor [10] and Cordeiro et al. [11] gave the research of the Gamma-Lomax distribution and the Poisson-Lomax distribution. Almetwally and Almongy [12] gave the parameter estimation of power Lomax distribution as well as the stressstrength model. 


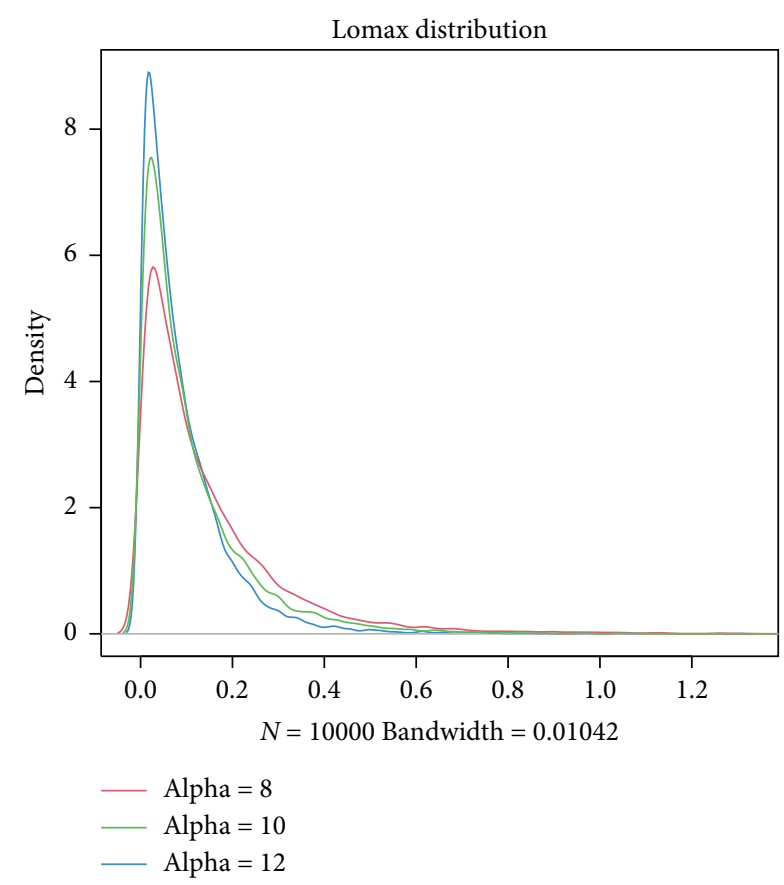

FIGURE 1: PDF of Lomax distribution with different values of $\alpha$.

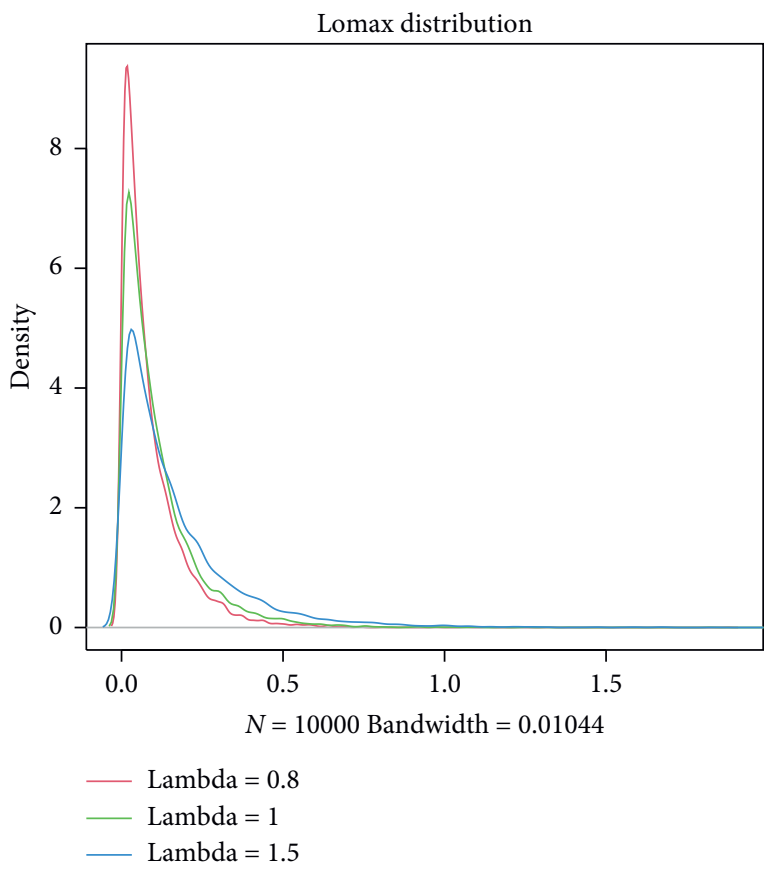

FIGURE 2: PDF of Lomax distribution with different values of $\lambda$.

In this paper, we deal with the shape parameter $\alpha$ of Lomax distribution and its reliability function $R(t)$ under the Type-I HCS.

\section{Bayesian Estimation of $\alpha$}

Usually in life test experiments, considering the cost, material resources, and time constraints, it may not be possible to fully observe the failure time of all products. In these cases,

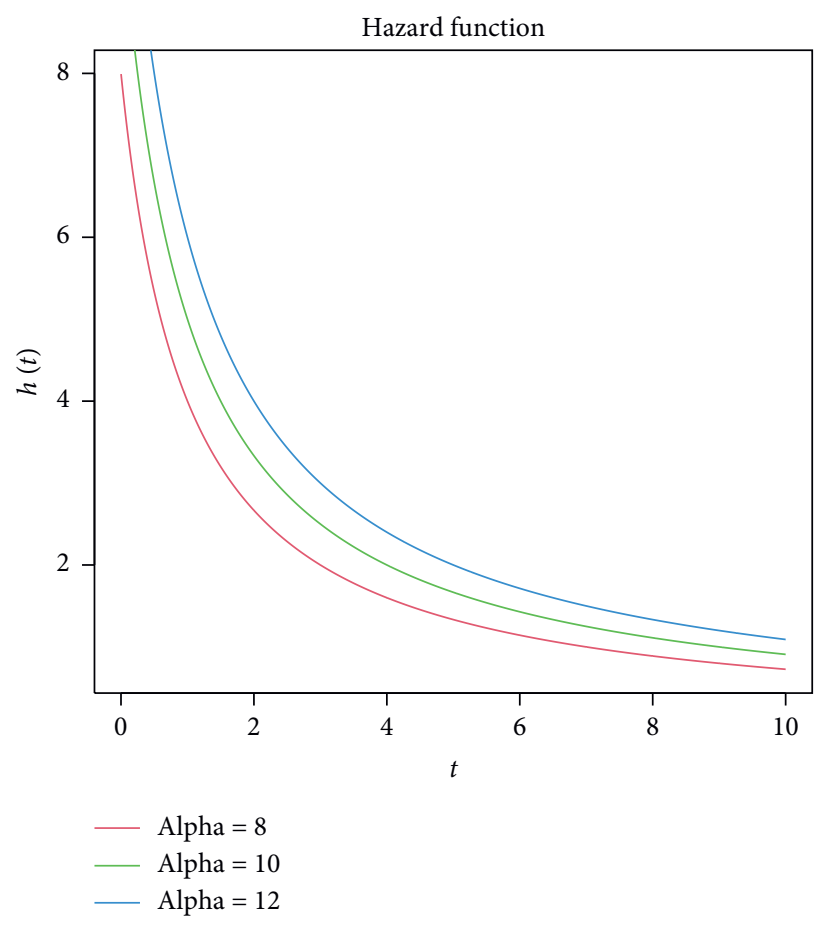

Figure 3: $h(t)$ of Lomax distribution with different values of $\alpha$.

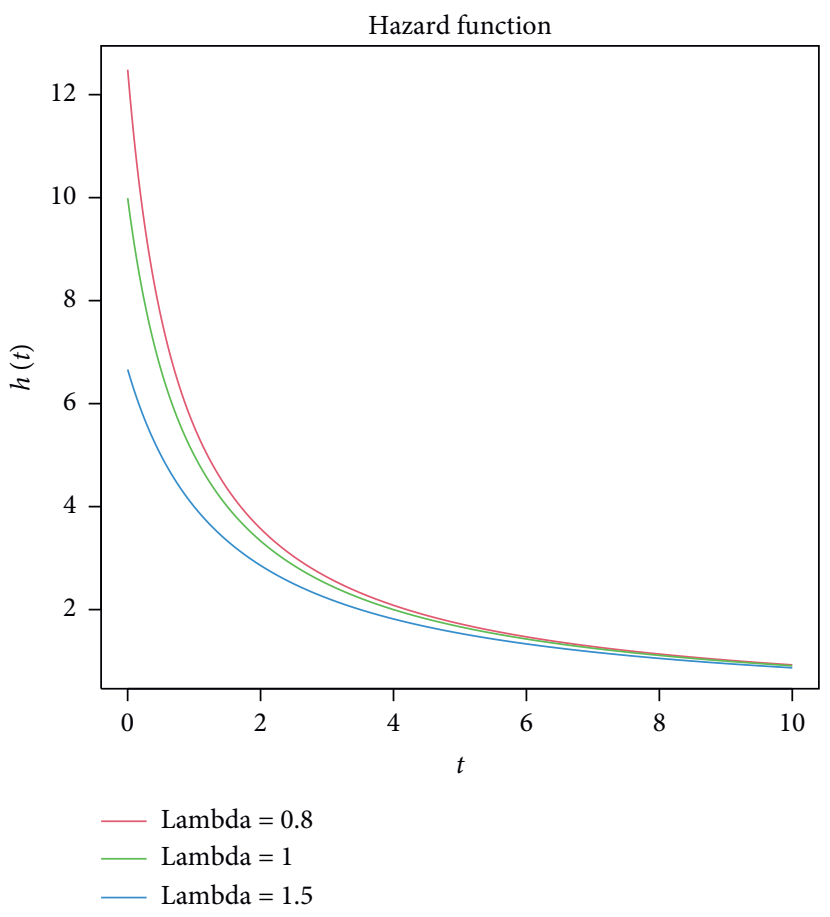

Figure 4: $h(t)$ of Lomax distribution with different values of $\lambda$.

the sample examined plays a very important role in the experiment. Ashour and Abdelfattah [13] presented more details about the estimation of the parameter of the Lomax distribution under HCS. A variety of analytical methods under incomplete sample conditions have been studied. Type-I HCS and Type-II HCS are two commonly used modes and are all designated to delete some samples when 
the life test comes to an end. Generalized Type-I HCS defines $T_{*}=\min \left\{T, X_{k: n}\right\}$ to ensure that the specified time is the upper limit of the time. There is a certain termination time for the experiment. But when the life of samples is longer and the number of failed items is comparatively small, failed samples will be insufficient. Due to time constraints, Type-I HCS is more suitable when time is limited.

Under generalized Type-I HCS, $X_{1: n}, \ldots, X_{n: n}$ are samples obeying Lomax distribution. We give the likelihood function for the following three cases by

$$
L(\alpha, \lambda)= \begin{cases}{\left[1-F\left(x_{k: n}\right)\right]^{n-k} \frac{n !}{(n-k) !} \prod_{i=1}^{D} f\left(x_{i: n}\right),} & D=0, \ldots, k-1, \\ {[1-F(T)]^{n-D} \frac{n !}{(n-D) !} \prod_{i=1}^{D} f\left(x_{i: n}\right),} & D=k, \ldots, r-1, \\ {\left[1-F\left(x_{r: n}\right)\right]^{n-r} \frac{n !}{(n-r) !} \prod_{i=1}^{r} f\left(x_{i: n}\right),} & D=r,\end{cases}
$$

where $\quad 0<X_{1: n}<\cdots<X_{k: n}<\quad \cdots<X_{d: n}<T<\cdots$ $<X_{r: n}<\cdots<X_{n: n}$. From (1), (2), and (7), we can conclude that the likelihood function is related to $\alpha$ and $\lambda$. When $\lambda$ is a fixed value, it is a function of $\alpha$, denoted by $L(\alpha)$. The function expression is as follows

$$
L(\alpha, \lambda)=\left\{\begin{array}{l}
{\left[\left(1+\frac{x_{k: n}}{\lambda}\right)^{-\alpha}\right]^{n-k} \frac{n !}{(n-k) !} \prod_{i=1}^{k}\left[\frac{\alpha}{\lambda}\left(1+\frac{x_{i: n}}{\lambda}\right)^{-(\alpha+1)}\right], \quad D=0, \ldots, k-1,} \\
{\left[\left(1+\frac{T}{\lambda}\right)^{-\alpha}\right]^{n-D} \frac{n !}{(n-D) !} \prod_{i=1}^{D}\left[\frac{\alpha}{\lambda}\left(1+\frac{x_{i: n}}{\lambda}\right)^{-(\alpha+1)}\right], \quad D=k, \ldots, r-1,} \\
{\left[\left(1+\frac{x_{r: n}}{\lambda}\right)^{-\alpha}\right]^{n-r} \frac{n !}{(n-r) !} \prod_{i=1}^{r}\left[\frac{\alpha}{\lambda}\left(1+\frac{x_{i: n}}{\lambda}\right)^{-(\alpha+1)}\right], \quad D=r .}
\end{array}\right.
$$

We first study the Bayesian estimation of shape parameter $\alpha$. It is necessary to determine the prior distribution whose function domain is consistent with the range of the unknown parameter $\alpha$. Because $\alpha>0, \lambda>0$, it is assumed that the prior PDF is shown as follows:

$$
\pi(\alpha)=\frac{b^{a}}{\Gamma(a)} \alpha^{a-1} e^{-b \alpha} .
$$

This is also the density function of gamma distribution. When $\lambda$ is fixed, and $x=\left(x_{1: n}, \ldots, x_{n: n}\right)$, from (8) and (9), the posterior PDF of $\alpha$ is given as

$$
\pi(\alpha \mid x)=\frac{L(\alpha) \pi(\alpha)}{\int_{0}^{+\infty} L(\alpha) \pi(\alpha) \mathrm{d} \alpha} .
$$

So, we can get the posterior PDF of $\alpha$ as

$$
\pi(\alpha \mid x)= \begin{cases}K^{-1}\left[\left(1+\frac{x_{k: n}}{\lambda}\right)^{-\alpha}\right]^{n-k} \alpha^{a+k-1} e^{-b \alpha} \prod_{i=1}^{k}\left(1+\frac{x_{i: n}}{\lambda}\right)^{-(\alpha+1)}, & D=0, \ldots, k-1, \\ K^{-1}\left[\left(1+\frac{T}{\lambda}\right)^{-\alpha}\right]^{n-D} \alpha^{a+D-1} e^{-b \alpha} \prod_{i=1}^{D}\left(1+\frac{x_{i: n}}{\lambda}\right)^{-(\alpha+1)}, & D=k, \ldots, r-1, \\ K^{-1}\left[\left(1+\frac{x_{r: n}}{\lambda}\right)^{-\alpha}\right]^{n-r} \alpha^{a+r-1} e^{-b \alpha} \prod_{i=1}^{r}\left(1+\frac{x_{i: n}}{\lambda}\right)^{-(\alpha+1)}, & D=r,\end{cases}
$$


in which $K$ is defined as

$$
K=\left\{\begin{array}{l}
\int_{0}^{+\infty}\left[\left(1+\frac{x_{k: n}}{\lambda}\right)^{-\alpha}\right]^{n-k} \alpha^{a+k-1} e^{-b \alpha} \prod_{i=1}^{k}\left(1+\frac{x_{i: n}}{\lambda}\right)^{-(\alpha+1)} \mathrm{d} \alpha, \quad D=0, \ldots, k-1, \\
\int_{0}^{+\infty}\left[\left(1+\frac{T}{\lambda}\right)^{-\alpha}\right]^{n-D} \alpha^{a+D-1} e^{-b \alpha} \prod_{i=1}^{D}\left(1+\frac{x_{i: n}}{\lambda}\right)^{-(\alpha+1)} \mathrm{d} \alpha, \quad D=k, \ldots, r-1, \\
\int_{0}^{+\infty}\left[\left(1+\frac{x_{r: n}}{\lambda}\right)^{-\alpha}\right]^{n-r} \alpha^{a+r-1} e^{-b \alpha} \prod_{i=1}^{r}\left(1+\frac{x_{i: n}}{\lambda}\right)^{-(\alpha+1)} \mathrm{d} \alpha, \quad D=r .
\end{array}\right.
$$

Under SEL function, from (11), the Bayesian estimation, $\widehat{\alpha}_{\mathrm{BS}}$, of $\alpha$ is defined as

$$
\begin{aligned}
\widehat{\alpha}_{\mathrm{BS}} & =E[\alpha \mid x] \\
& =\int_{0}^{+\infty} \alpha \pi(\alpha \mid x) \mathrm{d} \alpha \\
& =\left\{\begin{array}{l}
K^{-1} \int_{0}^{+\infty}\left[\left(1+\frac{x_{k: n}}{\lambda}\right)^{-\alpha}\right]^{n-k} \alpha^{a+k} e^{-b \alpha} \prod_{i=1}^{k}\left(1+\frac{x_{i: n}}{\lambda}\right)^{-(\alpha+1)} \mathrm{d} \alpha, \quad D=0, \ldots, k-1, \\
K^{-1} \int_{0}^{+\infty}\left[\left(1+\frac{T}{\lambda}\right)^{-\alpha}\right]^{n-D} \alpha^{a+D} e^{-b \alpha} \prod_{i=1}^{D}\left(1+\frac{x_{i: n}}{\lambda}\right)^{-(\alpha+1)} \mathrm{d} \alpha, \quad D=k, \ldots, r-1, \\
K^{-1} \int_{0}^{+\infty}\left[\left(1+\frac{x_{r: n}}{\lambda}\right)^{-\alpha}\right]^{n-r} \alpha^{a+r} e^{-b \alpha} \prod_{i=1}^{r}\left(1+\frac{x_{i: n}}{\lambda}\right)^{-(\alpha+1)} \mathrm{d} \alpha, \quad D=r .
\end{array}\right.
\end{aligned}
$$

Next, we take the LINEX function as the loss function. Wei et al. [14] discussed more about the composite LINEX loss of symmetry. Han [15] gave the E-Bayesian estimation under the LINEX loss function. This function is also useful in calculating the expectation, but in a different way. Therefore, we can give the Bayesian estimation, $\widehat{\alpha}_{\mathrm{BL}}$, of $\alpha$ as

$$
\begin{aligned}
\widehat{\alpha}_{\mathrm{BL}} & =\frac{-1}{h} \ln E\left[e^{-h \alpha} \mid x\right] \\
& =\frac{-1}{h} \ln \int_{0}^{+\infty} e^{-h \alpha} \pi(\alpha \mid x) \mathrm{d} \alpha \\
& =\left\{\begin{array}{l}
\frac{-1}{h} \ln \int_{0}^{+\infty} \frac{\alpha^{a+k-1}}{K} e^{-(h+b) \alpha}\left[\left(1+\frac{x_{k: n}}{\lambda}\right)^{-\alpha}\right]^{n-k} \prod_{i=1}^{k}\left(1+\frac{x_{i: n}}{\lambda}\right)^{-(\alpha+1)} \mathrm{d} \alpha, \quad D=0, \ldots, k-1, \\
\frac{-1}{h} \ln \int_{0}^{+\infty} \frac{\alpha^{a+D-1}}{K} e^{-(h+b) \alpha}\left[\left(1+\frac{T}{\lambda}\right)^{-\alpha}\right]^{n-D} \prod_{i=1}^{D}\left(1+\frac{x_{i: n}}{\lambda}\right)^{-(\alpha+1)} \mathrm{d} \alpha, \quad D=k, \ldots, r-1, \\
\frac{-1}{h} \ln \int_{0}^{+\infty} \frac{\alpha^{a+r-1}}{K} e^{-(h+b) \alpha}\left[\left(1+\frac{x_{r: n}}{\lambda}\right)^{-\alpha}\right]^{n-r} \prod_{i=1}^{r}\left(1+\frac{x_{i: n}}{\lambda}\right)^{-(\alpha+1)} \mathrm{d} \alpha, \quad D=r .
\end{array}\right.
\end{aligned}
$$


Similarly, under SEL function, we study the Bayesian estimation of reliability function, $\widehat{R}(t)_{\mathrm{BS}}$. It can be calculated in the same as the parameter estimation. So, $\widehat{R}(t)_{\mathrm{BS}}$ is given as

$$
\begin{aligned}
\widehat{R}(t)_{\mathrm{BS}}= & E[R(t) \mid x] \\
& =\int_{0}^{+\infty} \pi(\alpha \mid x) R(t) \mathrm{d} \alpha \\
& =\left\{\begin{array}{l}
K^{-1} \int_{0}^{+\infty} \alpha^{a+k-1} e^{-b \alpha}\left[\left(1+\frac{x_{k: n}}{\lambda}\right)^{-\alpha}\right]^{n-k}\left(1+\frac{t}{\lambda}\right)^{-\alpha} \times \prod_{i=1}^{k}\left(1+\frac{x_{i: n}}{\lambda}\right)^{-(\alpha+1)} \mathrm{d} \alpha, \quad D=0, \ldots, k-1, \\
K^{-1} \int_{0}^{+\infty} \alpha^{a+D-1} e^{-b \alpha}\left[\left(1+\frac{T}{\lambda}\right)^{-\alpha}\right]^{n-D}\left(1+\frac{t}{\lambda}\right)^{-\alpha} \times \prod_{i=1}^{D}\left(1+\frac{x_{i: n}}{\lambda}\right)^{-(\alpha+1)} \mathrm{d} \alpha, \quad D=k, \ldots, r-1, \\
K^{-1} \int_{0}^{+\infty} \alpha^{a+r-1} e^{-b \alpha}\left[\left(1+\frac{x_{r: n}}{\lambda}\right)^{-\alpha}\right]^{n-r}\left(1+\frac{t}{\lambda}\right)^{-\alpha} \times \prod_{i=1}^{r}\left(1+\frac{x_{i: n}}{\lambda}\right)^{-(\alpha+1)} \mathrm{d} \alpha, \quad D=r .
\end{array}\right.
\end{aligned}
$$

In the same way, we can use the method under the LINEX loss function. This method is also useful in calculating expectation, but with a different function. The Bayesian estimation, $\widehat{R}(t)_{\mathrm{BL}}$, is calculated as

$$
\begin{aligned}
\widehat{R}(t)_{\mathrm{BL}}= & \frac{-1}{h} \ln E\left[e^{-h R(t)} \mid x\right] \\
& =\frac{-1}{h} \ln \int_{0}^{+\infty} e^{-h R(t)} \pi(\alpha \mid x) \mathrm{d} \alpha \\
& \left\{\begin{array}{l}
\frac{-1}{h} \ln \int_{0}^{+\infty}\left[\left(1+\frac{x_{k: n}}{\lambda}\right)^{-\alpha}\right]^{n-k} K^{-1} \alpha^{a+k-1} \exp \left\{-h\left(1+\frac{t}{\lambda}\right)^{-\alpha}-b \alpha\right\} \times \prod_{i=1}^{k}\left(1+\frac{x_{i: n}}{\lambda}\right)^{-(\alpha+1)} \mathrm{d} \alpha, \quad D=0, \ldots, k-1, \\
\frac{-1}{h} \ln \int_{0}^{+\infty}\left[\left(1+\frac{T}{\lambda}\right)^{-\alpha}\right]^{n-D} K^{-1} \alpha^{a+D-1} \exp \left\{-h\left(1+\frac{t}{\lambda}\right)^{-\alpha}-b \alpha\right\} \times \prod_{i=1}^{D}\left(1+\frac{x_{i: n}}{\lambda}\right)^{-(\alpha+1)} \mathrm{d} \alpha, \quad D=k, \ldots, r-1, \\
\frac{-1}{h} \ln \int_{0}^{+\infty}\left[\left(1+\frac{x_{r: n}}{\lambda}\right)^{-\alpha}\right]^{n-r} K^{-1} \alpha^{a+r-1} \exp \left\{-h\left(1+\frac{t}{\lambda}\right)^{-\alpha}-b \alpha\right\} \times \prod_{i=1}^{r}\left(1+\frac{x_{i: n}}{\lambda}\right)^{-(\alpha+1)} \mathrm{d} \alpha, \quad D=r .
\end{array}\right.
\end{aligned}
$$

\section{E-Bayesian Estimation of $\alpha$}

In order to be sure that $\pi(\alpha)$ is a prior distribution of $\alpha$, the selected $a$ and $b$, the prior parameters, need some restrictions. For $\alpha, \pi(\alpha)$ must be a decreasing function. Referring to Han [16], we can get more details. To determine the value range of $a$ and $b$, we need to calculate $\mathrm{d} \pi(\alpha) / \mathrm{d} \alpha$, the derivative of $\pi(\alpha)$. From the calculation, we get the following results:

$$
\frac{\mathrm{d} \pi(\alpha)}{\mathrm{d} \alpha}=\frac{b^{a}}{\Gamma(a)} \alpha^{a-2} e^{-b \alpha}\{a-1-b \alpha\}, \quad 0<a<1, b>0 .
$$

Being a decreasing function, the prior PDF should be guaranteed that $\mathrm{d} \pi(\alpha) / \mathrm{d} \alpha<0$, which equals to $a-1-b \alpha<0$. So, $0<a<1, b>0$ is a solution set of the inequality. We suppose that the hyperparameters $a$ and $b$ are independent, so we are able to give the bivariate PDF of $a$ and $b$ as

$$
\pi(a, b)=\pi_{1}(a) \pi_{2}(b), \quad 0<a<1, b>0 .
$$

For the parameter $\alpha$, when the Bayesian estimation is assumed to be $\widehat{\alpha}_{B}(a, b)$, the E-Bayesian estimation $\widehat{\alpha}_{\mathrm{EB}}$ is

$$
\widehat{\alpha}_{\mathrm{EB}}=E\left[\widehat{\alpha}_{B} \mid x\right]=\iint_{Q} \widehat{\alpha}_{B}(a, b) \pi(a, b) \mathrm{d} a \mathrm{~d} b,
$$


and for $R(t)$, when the Bayesian estimation is assumed to be $\widehat{R}(t)$, the E-Bayesian estimation $\widehat{R}(t)_{\mathrm{EB}}$ is

$$
\widehat{R}(t)_{\mathrm{EB}}=E\left[\widehat{R}(t)_{B} \mid x\right]=\iint_{Q} \widehat{R}(t, a, b)_{B} \pi(a, b) \mathrm{d} a \mathrm{~d} b,
$$

in which $Q=\{(a, b) \mid 0<a<1,0<b<c\}$.

If $X_{1: n}, \ldots, X_{k: n}$ obey the Lomax distribution (1), we can get (8) which is the likelihood function of $\alpha$, and (10) is the prior density function. For $(10), \pi(\alpha \mid a, b)$ is determined not only by $\alpha$ but also by $a$ and $b$. Referring to Han [6], $\pi(a, b)$ can be defined as

$$
\pi(a, b)=\frac{1}{c}, \quad 0<a<1,0<b<c .
$$

Han [17] first addressed the E-Bayesian estimation. We get the E-Bayesian estimation by computing the expectation again for the Bayesian estimation which is related to $a$ and $b$. The E-Bayesian estimation of $\alpha$ under SEL function is got according to (13), (19), and (21) as

$$
\widehat{\alpha}_{\mathrm{EBS}}=\iint_{\mathrm{Q}} \widehat{\alpha}_{\mathrm{BS}}(a, b) \pi(a, b) \mathrm{d} a \mathrm{~d} b, \quad 0<a<1,0<b<c .
$$

For LINEX loss function, the E-Bayesian estimation of $\alpha$ is got according to (14), (19), and (21) as

$$
\widehat{\alpha}_{\mathrm{EBL}}=\iint_{Q} \widehat{\alpha}_{\mathrm{BL}}(a, b) \pi(a, b) \mathrm{d} a \mathrm{~d} b, \quad 0<a<1,0<b<c .
$$

Under the SEL function, $R(t)$ can be estimated by EBayesian estimation as well. According to (21), (15), and (20), we get the E-Bayesian estimation of $R(t)$. When $t$ is constant, the primitive function is a function of $a$ and $b$ only. The estimated value of the function is

$$
\widehat{R}(t)_{\mathrm{EBS}}=\iint_{Q} \widehat{R}(t)_{\mathrm{BS}}(a, b) \pi(a, b) \mathrm{d} a \mathrm{~d} b, \quad 0<a<1,0<b<c .
$$

The same method is also suitable for the situation under the LINEX loss function. According to (16), (20), and (21), we get the E-Bayesian estimation of $R(t)$. When $t$ is constant, the primitive function is also a function of $a$ and $b$ only. The estimated value of the function is

$$
\widehat{R}(t)_{\mathrm{EBL}}=\iint_{Q} \widehat{R}(t)_{\mathrm{BL}}(a, b) \pi(a, b) \mathrm{d} a \mathrm{~d} b, \quad 0<a<1,0<b<c .
$$

Next, we study the mean square error of the estimated value in order to judge the validity of the estimation. The proposed time of E-Bayesian estimation is short, and the research results we have already obtained are also less. For the E-Bayesian estimation, the research on the mean square error is still in a relatively new area. The E-MSE is proposed by Han [4] and Han [5] in the case of the two hyperparameters and one hyperparameter. For more information about E-MSE, we can refer to these two articles. We specify that E-MSE means the expected mean square error, from which we get the MSE of $\widehat{\alpha}_{\mathrm{BS}}(a, b)$ under SEL function which is

$$
\operatorname{MSE}\left[\widehat{\alpha}_{\mathrm{BS}}(a, b)\right]=E\left\{\left[\alpha-\widehat{\alpha}_{\mathrm{BS}}(a, b)\right]^{2} \mid x\right\} .
$$

Under LINEX loss function, we can also get the MSE of $\widehat{\alpha}_{\mathrm{BL}}(a, b)$ as

$$
\operatorname{MSE}\left[\widehat{\alpha}_{\mathrm{BL}}(a, b)\right]=E\left\{\left[\alpha-\widehat{\alpha}_{\mathrm{BL}}(a, b)\right]^{2} \mid x\right\} .
$$

Under SEL function, we can get the expectation of $\operatorname{MSE}\left[\widehat{\alpha}_{\mathrm{BS}}(a, b)\right]$ which is $E-\operatorname{MSE}\left[\widehat{\alpha}_{\mathrm{BS}}(a, b)\right]$ as follows:

$$
E-\operatorname{MSE}\left[\widehat{\alpha}_{\mathrm{BS}}(a, b)\right]=\iint_{\mathrm{Q}} \operatorname{MSE}\left[\widehat{\alpha}_{\mathrm{BS}}(a, b)\right] \pi(a, b) \mathrm{d} a \mathrm{~d} b,
$$

and under LINEX loss function, $E-\operatorname{MSE}\left[\widehat{\alpha}_{\mathrm{BL}}(a, b)\right]$ is

$$
E-\operatorname{MSE}\left[\widehat{\alpha}_{\mathrm{BL}}(a, b)\right]=\iint_{\mathrm{Q}} \operatorname{MSE}\left[\widehat{\alpha}_{\mathrm{BL}}(a, b)\right] \pi(a, b) \mathrm{d} a \mathrm{~d} b .
$$

Then, with these formulas, we are able to analyze $\widehat{\alpha}_{\mathrm{EBS}}$, $\widehat{\alpha}_{\mathrm{EBL}}, \widehat{R}(t)_{\mathrm{EBS}}, \widehat{R}(t)_{\mathrm{EBL}}$, and their MSE in detail.

\section{Calculation Results}

When we do not know the shape parameter but with the scale parameter, according to (8), the likelihood function follows the rules:

$$
L(x \mid \alpha) \propto \alpha^{n} \exp (-\alpha M),
$$

in which

$$
M= \begin{cases}\sum_{i=1}^{k} \ln \left(1+\frac{x_{i: n}}{\lambda}\right)+(n-k) \ln \left(1+\frac{x_{i: k}}{\lambda}\right), & D=0, \ldots, k-1, \\ \sum_{i=1}^{D} \ln \left(1+\frac{x_{i: n}}{\lambda}\right)+(n-D) \ln \left(1+\frac{T}{\lambda}\right), & D=k, \ldots, r-1, \\ \sum_{i=1}^{r} \ln \left(1+\frac{x_{i: n}}{\lambda}\right)+(n-r) \ln \left(1+\frac{x_{r: n}}{\lambda}\right), & D=r .\end{cases}
$$


We define that $l$ is an integer and

$$
l= \begin{cases}k, & D=0, \ldots, k-1, \\ D, & D=k, \ldots, r-1, \\ r, & D=r .\end{cases}
$$

4.1. The E-MSE of $\widehat{\alpha}_{E B}$ under SEL Function. Under SEL function, $L_{1}(\alpha, \delta)=(\alpha-\delta)^{2}$. We can calculate $\hat{\alpha}_{\mathrm{BS}}$, the Bayesian estimation of $\alpha$, directly. So, $\widehat{\alpha}_{\mathrm{BS}}$ is given by

$$
\begin{aligned}
\widehat{\alpha}_{\mathrm{BS}} & =E[\alpha \mid x]=\int_{0}^{+\infty} \alpha \pi(\alpha \mid x) \mathrm{d} \alpha \\
& =\frac{\int_{0}^{+\infty} \alpha L(\alpha) \pi(\alpha) \mathrm{d} \alpha}{\int_{0}^{+\infty} L(\alpha) \pi(\alpha) \mathrm{d} \alpha}=\frac{\int_{0}^{+\infty} \alpha^{a+l} e^{-(b+M) \alpha} \mathrm{d} \alpha}{\int_{0}^{+\infty} \alpha^{a+l-1} e^{-(b+M) \alpha} \mathrm{d} \alpha} \\
& =\frac{\left(\Gamma(a+l+1) /(b+M)^{a+l+1}\right)}{\left(\Gamma(a+l) /(b+M)^{a+l}\right)}=\frac{a+l}{b+M} .
\end{aligned}
$$
(33) as

According to Han [6], $\widehat{\alpha}_{\mathrm{EBS}}$ is got from (21), (22), and

$$
\begin{aligned}
\widehat{\alpha}_{\mathrm{EBS}} & =\iint_{Q} \widehat{\alpha}_{\mathrm{BS}}(a, b) \pi(a, b) \mathrm{d} a \mathrm{~d} b \\
& =\iint_{\mathrm{Q}} \frac{a+l}{b+M} \frac{1}{c} \mathrm{~d} a \mathrm{~d} b=\frac{1}{c} \int_{0}^{1}(a+l) \mathrm{d} a \int_{0}^{c} \frac{1}{b+M} \mathrm{~d} b=\frac{2 l+1}{2 c} \ln \left(\frac{M+c}{M}\right) .
\end{aligned}
$$

From (34), we can know the E-Bayesian estimation of $\alpha$. The final result can be simplified to a formula as

$$
\widehat{\alpha}_{\mathrm{EBS}}=\frac{2 l+1}{2 c} \ln \left(\frac{M+c}{M}\right) \text {. }
$$

Under SEL function, $\operatorname{MSE}\left[\widehat{\alpha}_{\mathrm{BS}}(a, b)\right]$ is got from (26) and (33) as

$$
\operatorname{MSE}\left[\widehat{\alpha}_{\mathrm{BS}}(a, b)\right]=E\left\{\left[\alpha-\widehat{\alpha}_{\mathrm{BS}}(a, b)\right]^{2} \mid x\right\} .
$$

According to the proof procedure in Han [6], the posterior distribution of $\alpha$ is $\operatorname{Gamma}(l+a, M+b)$. In gamma distribution, we can directly use variance as the mean square error of estimation. The expression of variance is $\operatorname{Var}(\alpha \mid x)=(l+a) /(M+b)^{2}$. The Bayesian estimation of $\alpha$ is $\widehat{\alpha}_{\mathrm{BS}}=(a+l) /(b+M)$. So, the MSE of $\widehat{\alpha}_{\mathrm{BS}}$ is

$$
\operatorname{MSE}\left[\widehat{\alpha}_{\mathrm{BS}}(a, b)\right]=E\left\{\left[\alpha-\widehat{\alpha}_{\mathrm{BS}}(a, b)\right]^{2} \mid x\right\}=\operatorname{Var}(\alpha \mid x)=\frac{l+a}{(M+b)^{2}} \text {. }
$$

$\pi(a, b)$ is obtained from (34), then the $E-\operatorname{MSE}\left(\widehat{\alpha}_{\mathrm{EBS}}\right)$ is given as

$$
\begin{aligned}
E-\operatorname{MSE}\left(\widehat{\alpha}_{\mathrm{EBS}}\right) & =\iint_{Q} \operatorname{MSE}\left[\widehat{\alpha}_{\mathrm{BS}}(a, b)\right] \pi(a, b) \mathrm{d} a \mathrm{~d} b \\
& =\iint_{Q} \frac{l+a}{(M+b)^{2}} \frac{1}{c} \mathrm{~d} a \mathrm{~d} b=\frac{1}{c} \int_{0}^{1}(a+l) \mathrm{d} a \int_{0}^{c} \frac{1}{(b+M)^{2}} \mathrm{~d} b=\frac{2 l+1}{2 M(M+c)},
\end{aligned}
$$

from which we can get the E-MSE of $\widehat{\alpha}_{\mathrm{EBS}}$ which is

$$
E-\operatorname{MSE}\left(\widehat{\alpha}_{\mathrm{EBS}}\right)=\frac{2 l+1}{2 M(M+c)} .
$$

4.2. The E-MSE of $\widehat{\alpha}_{E B}$ under LINEX Function. The derivation method is the same as that under SEL function. Under LINEX function, the calculation process of $\widehat{\alpha}_{\mathrm{BL}}$ is

$$
\begin{aligned}
\widehat{\alpha}_{\mathrm{BL}} & =\frac{-1}{h} \ln E\left[e^{-h \alpha} \mid x\right]=\frac{-1}{h} \ln \int_{0}^{+\infty} e^{-h \alpha} \pi(\alpha \mid x) \mathrm{d} \alpha \\
& =\frac{-1}{h} \ln \frac{\int_{0}^{+\infty} e^{-h \alpha} L(\alpha) \pi(\alpha) \mathrm{d} \alpha}{\int_{0}^{+\infty} L(\alpha) \pi(\alpha) \mathrm{d} \alpha}=\frac{-1}{h} \ln \frac{\int_{0}^{+\infty} \alpha^{a+l-1} e^{-(b+M+h) \alpha} \mathrm{d} \alpha}{\int_{0}^{+\infty} \alpha^{a+l-1} e^{-(b+M) \alpha} \mathrm{d} \alpha} \\
& =\frac{-1}{h} \ln \frac{\left(\Gamma(a+l) /(b+M+h)^{a+l}\right)}{\left(\Gamma(a+l) /(b+M)^{a+l}\right)}=\frac{-1}{h} \ln \left(\frac{b+M}{b+M+h}\right)^{a+l} \\
& =-\frac{a+l}{h} \ln \frac{b+M}{b+M+h} .
\end{aligned}
$$


In the same way, under LINEX function, we get the EBayesian estimation of $\alpha$ from (21), (23), and (40) as

$$
\begin{aligned}
\widehat{\alpha}_{\mathrm{EBL}} & =\iint_{Q} \widehat{\alpha}_{\mathrm{BL}}(a, b) \pi(a, b) \mathrm{d} a \mathrm{~d} b \\
& =-\frac{1}{h c} \iint_{Q}(a+l) \ln \frac{b+M}{b+M+h} \mathrm{~d} a \mathrm{~d} b=-\frac{1}{h c} \int_{0}^{1}(a+l) \mathrm{d} a \int_{0}^{c} \ln \frac{b+M}{b+M+h} \mathrm{~d} b=-\frac{2 l+1}{2 h c} \int_{0}^{c} \ln \frac{b+M}{b+M+h} \mathrm{~d} b
\end{aligned}
$$

from which we can get the E-Bayesian estimation of $\alpha$ as

$$
\widehat{\alpha}_{\mathrm{EBL}}=-\frac{2 l+1}{2 h c} \int_{0}^{c} \ln \frac{b+M}{b+M+h} \mathrm{~d} b .
$$

Under LINEX function, we get the $\operatorname{MSE}\left[\widehat{\alpha}_{\mathrm{BL}}(a, b)\right]$ from (27) and (40) as

$$
\begin{aligned}
\operatorname{MSE}\left[\widehat{\alpha}_{\mathrm{BL}}(a, b)\right]= & E\left\{\left[\alpha-\widehat{\alpha}_{\mathrm{BL}}(a, b)\right]^{2} \mid x\right\} \\
= & E\left(\alpha^{2} \mid x\right)-2 \widehat{\alpha}_{\mathrm{BL}}(a, b) E(\alpha \mid x)+\left[\widehat{\alpha}_{\mathrm{BL}}(a, b)\right]^{2} \\
= & \frac{(a+l+1)(a+l)}{(b+M)^{2}}+2 \frac{(a+l)^{2}}{h(b+M)} \ln \frac{b+M}{b+M+h} \\
& +\left(\frac{a+l}{h} \ln \frac{b+M}{b+M+h}\right)^{2} .
\end{aligned}
$$

$\pi(a, b)$ is given by $(21)$, then $E-\operatorname{MSE}\left(\widehat{\alpha}_{\mathrm{EBL}}\right)$ is

$$
\begin{aligned}
E-\operatorname{MSE}\left(\widehat{\alpha}_{\mathrm{EBL}}\right)= & \iint_{Q} \operatorname{MSE}\left[\widehat{\alpha}_{\mathrm{BL}}(a, b)\right] \pi(a, b) \mathrm{d} a \mathrm{~d} b \\
= & \int_{0}^{1}(a+l)(a+l+1) \mathrm{d} a \int_{0}^{c} \ln \frac{1}{(b+M)^{2}} \mathrm{~d} b \\
& +\frac{2}{h} \int_{0}^{1}(a+l)^{2} \mathrm{~d} a \int_{0}^{c} \frac{1}{(b+M)} \ln \frac{b+M}{b+M+h} \mathrm{~d} b \\
& +\frac{1}{h^{2}} \int_{0}^{1}(a+l)^{2} \mathrm{~d} a \int_{0}^{c}\left(\ln \frac{b+M}{b+M+h}\right)^{2} \mathrm{~d} b,
\end{aligned}
$$

from which we can get the E-MSE of $\widehat{\alpha}_{\mathrm{EBL}}$.

Having the formulas mentioned above, we first calculate the Bayesian estimation of $\alpha$ by calculus. Then, by dealing with Bayesian estimation, we get the E-Bayesian estimation.
The MSE of Bayesian estimation is computed as well. Finally, according to the MSE, we can get the E-MSE.

\section{MCMC Method}

For the estimators that are not easy to calculate directly by calculus, we generate the Markov chain by the Metropolis-Hastings $(\mathrm{MH})$ algorithm. With the Markov chain, we can calculate the Bayesian and E-Bayesian estimations of $\alpha$ and $R(t)$. This method is one of the Markov chain Monte Carlo (MCMC) methods, which is widely used and effective. We apply the $\mathrm{MH}$ algorithm to generate posterior samples of $\alpha$ from a complete conditional posterior PDF.

In the $\mathrm{MH}$ algorithm, we need to find a proposal distribution similar to the original distribution as the distribution function of the generated samples. Due to the high complexity of the conditional posterior PDF of $\alpha$, it is not convenient for us to find a similar distribution. So, the MCMC algorithm is introduced with the normal proposal distribution. From the normal proposal distribution, we are able to obtain random samples of $\alpha$.

According to the calculation formulas in Section 4, we can calculate the estimated value and mean square error under different conditions, by taking them as the expectation and variance of a normal distribution. Then, we take this normal distribution as the potential distribution to generate a Markov chain. At the same time, according to the calculation formula in Section 4, we can delete some samples that are too large or too small from the samples of the Markov chain and keep the maximum $\left(\alpha_{N[1-(\gamma / 2)]}\right)$ and minimum values $\left(\alpha_{N[\gamma / 2]}\right)$ of the remaining samples. Taking $\alpha_{N[\gamma / 2]}$ and $\alpha_{N[\gamma / 2]}$ as the endpoints of the CRI, and defining $\alpha_{N[\gamma / 2]}-\alpha_{N[\gamma / 2]}$ as the length of the CRI, we get the complete conditional posterior PDF of $\alpha$ from (11), which is shown as follows:

$$
\pi^{*}(\alpha \mid x)= \begin{cases}\alpha^{a+k-1} e^{-b \alpha}\left[\left(1+\frac{x_{k: n}}{\lambda}\right)^{-\alpha}\right]^{n-k} \prod_{i=1}^{k}\left(1+\frac{x_{i: n}}{\lambda}\right)^{-(\alpha+1)}, & D=0, \ldots, k-1, \\ \alpha^{a+D-1} e^{-b \alpha}\left[\left(1+\frac{T}{\lambda}\right)^{-\alpha}\right]^{n-D} \prod_{i=1}^{D}\left(1+\frac{x_{i: n}}{\lambda}\right)^{-(\alpha+1)}, & D=k, \ldots, r-1, \\ \alpha^{a+r-1} e^{-b \alpha}\left[\left(1+\frac{x_{r: n}}{\lambda}\right)^{-\alpha}\right]^{n-r} \prod_{i=1}^{r}\left(1+\frac{x_{i: n}}{\lambda}\right)^{-(\alpha+1)}, & D=r .\end{cases}
$$


Hastings [18] and Cowles and Carlin [19] can be referred to for more details, from which we can propose the algorithm as follows:

(i) Begin this process with $\alpha^{(0)}$, an initial guess $\left(\alpha^{(0)}=\alpha_{\mathrm{MLE}}\right)$.

(ii) From a proposal distribution, generate $\alpha^{(*)}$ at iteration $j$. We can select a normal distribution in this step.

(iii) From $U(0,1)$, generate a random number $u$.

(iv) Compute the acceptance probability shown as

$$
r\left(\alpha^{(j-1)} \mid \alpha^{(*)}\right)=\min \left[\frac{\pi^{*}\left(\alpha^{(*)} \mid x\right)}{\pi^{*}\left(\alpha^{(j-1)} \mid x\right)}, 1\right] .
$$

(v) If $u<r$, let $\alpha^{(j)}=\alpha^{(*)}$, and if $u \geq r$, let $\alpha^{(j)}=\alpha^{(j-1)}$.

(vi) With the generated values of $\alpha^{(j)}$, use

$$
R^{(j)}(t)=\left(1+\frac{t}{\lambda}\right)^{-\alpha^{(j)}}
$$

to calculate the value of $R^{(j)}(t)$.

(vii) Repeat (iii-vi) $\mathrm{N}$ times and define that $j=1, \ldots, N$.

(viii) Under SEL function, $\widehat{\alpha}_{\mathrm{BS}}$ and $\widehat{R}_{\mathrm{BS}}(t)$ are given by

$$
\begin{aligned}
\widehat{\alpha}_{\mathrm{BS}} & =\frac{1}{N-M} \sum_{j=M+1}^{N} \alpha^{(j)}, \\
\widehat{R}_{\mathrm{BS}}(t) & =\frac{1}{N-M} \sum_{j=M+1}^{N} R^{(j)}(t) .
\end{aligned}
$$

$\mathrm{M}$ is a burn-in period which we can decide during the experiment.

(ix) Under LINEX loss function, $\widehat{\alpha}_{\mathrm{BL}}$ and $\widehat{R}_{\mathrm{BL}}(t)$ are given by

$$
\begin{gathered}
\widehat{\alpha}_{\mathrm{BL}}=-h^{-1} \ln \left[\frac{1}{N-M} \sum_{j=M+1}^{N} e^{-h \alpha^{(j)}}\right], \\
\widehat{R}_{\mathrm{BL}}(t)=-h^{-1} \ln \left[\frac{1}{N-M} \sum_{j=M+1}^{N} e^{-h R^{(j)}(t)}\right] .
\end{gathered}
$$

(x) With MCMC draws, we can get the $100(1-\gamma) \%$ CRIs. With the generated samples and two selected sample quantiles, $(\gamma / 2)$ and $(1-(\gamma / 2))$, we get the CRIs which are

$$
\begin{gathered}
\left(\alpha_{N[\gamma / 2]}, \alpha_{N[1-(\gamma / 2)]}\right), \\
\left(R(t)_{N[\gamma / 2]}, R(t)_{N[1-(\gamma / 2)]}\right),
\end{gathered}
$$

where $\mathrm{N}$ represents the number of draws.
5.1. Simulation Study. Some simulation results are presented in this subsection when the samples have different values of $n=50,80,120, r=40,60,90, k=30,40,60$, and $T=0.2,0.4$. We assume that $\lambda=1$ in (1).

(i) The generation of the parameter $\alpha$ and the hyperparameters $a$ and $b$ :

We generate random values of hyperparameters $a$ and $b$ from (21) when the value of $c$ is fixed, and from (9), which equals to the PDF of gamma distribution, we generate random parameters $\alpha$. The generated value of $\alpha$ is taken as the actual value, and the inverse function method is used.

(ii) The generation of generalized Type-I hybrid censored samples:

We first use a uniform distribution to generate the value of the probability function and then get the corresponding sample value from the probability function (2). With this method, we can get the samples by

$$
X=\left[(1-U)^{-(1 / \alpha)}-1\right] \lambda,
$$

in which the random number $U$ is generated from $U(0,1)$.

After generating samples, we keep nearly half of them as censored samples. We sort the generated samples from small to large and select the sample approaching the median as the $k$-th sample. Then, we choose a value close to $X_{1: n}+\left(X_{n: n}-\right.$ $\left.X_{1: n}\right) / 2$ or $X_{1: n}+3\left(X_{n: n}-X_{1: n}\right) / 4$ as the experimental time $T$ and choose a number between $n$ and $k$ as $r$. For the convenience of calculation, the values of $n, k, r$, and $T$ can be selected in a reasonable range. Then, we can calculate that $T_{*}=\min \left\{T, X_{k: n}\right\}$ and generate the generalized Type-I hybrid censored samples. This set of samples can be represented as set $\left\{X_{i: n} \mid X_{i: n}<T_{*}\right\}$.

With $\mathrm{MH}$ algorithm, a Markov chain that includes 11,000 samples of $\alpha$ is generated. As a burn-in period, the first 1000 observation samples are discarded. Under two types of loss functions, we conclude the Bayesian estimations of $\alpha$ from (48) and (50); with the values of $\alpha$, we generate and put all figures in Tables 1 and 2.

Under two loss functions, SEL and LINEX, we get the Bayesian estimations of $R(t)$ from (49) and (51) with the same methods and put them in Tables 3 and 4 . Then, we get all four sets of estimations.

The E-Bayesian estimations of $\alpha$ are computed from (34), (22), and (23) under two types of loss functions, SEL and LINEX. Under two loss functions, we get the E-Bayesian estimations of $R(t)$ from (41), (24), and (25). We use the MSE to compare the effectiveness of the estimations of $\alpha$ and $R(t)$, which are

$$
\begin{aligned}
\operatorname{MSE}(\widehat{\alpha}) & =\frac{1}{N-M} \sum_{j=M+1}^{N}\left(\widehat{\alpha}_{j}-\alpha\right)^{2}, \\
\operatorname{MSE}(\widehat{R}(t)) & =\frac{1}{N-M} \sum_{j=M+1}^{N}\left(\widehat{R}(t)_{j}-R(t)\right)^{2},
\end{aligned}
$$

so when $N=11000$ and $M=1000$, 
TABLE 1: $\widehat{\alpha}_{\mathrm{BS}}, \widehat{\alpha}_{\mathrm{EBS}}, \mathrm{MSE}, \mathrm{E}-\mathrm{MSE}$, and the lengths of 95\% CRIs of $\alpha$ under SEL functions when $c=1, h=1.5, b=0.15234, a=0.6119$, $\alpha=4.36534$, and $\lambda=1$.

\begin{tabular}{ccccccccc}
\hline$n$ & $(r, k)$ & $T$ & $\widehat{\alpha}_{\text {BS }}$ & MSE & Length & $\widehat{\alpha}_{\text {EBS }}$ & $E-$ MSE & Length \\
\hline \multirow{2}{*}{50} & \multirow{2}{*}{$(40,30)$} & 0.2 & 4.48532 & 0.66552 & 3.84958 & 4.25887 & 0.62027 & 3.55299 \\
& & 0.4 & 4.43912 & 0.51408 & 2.93756 & 4.25816 & 0.47574 & 2.86497 \\
\multirow{2}{*}{80} & \multirow{2}{*}{$(60,40)$} & 0.2 & 4.39555 & 0.43270 & 2.61467 & 4.24306 & 0.40352 \\
& & 0.4 & 4.43516 & 0.33360 & 2.06987 & 4.31603 & 0.31644 & 1.83822 \\
\hline \multirow{2}{*}{120} & \multirow{2}{*}{$(90,60)$} & 0.2 & 4.38541 & 0.29225 & 1.91534 & 4.28172 & 0.27916 & 1.70398 \\
& & 0.4 & 4.40285 & 0.21800 & 1.27407 & 4.32383 & 0.21049 & 1.21373 \\
\hline
\end{tabular}

TABLE 2: $\widehat{\alpha}_{\mathrm{BL}}, \widehat{\alpha}_{\mathrm{EBL}}, \mathrm{MSE}, \mathrm{E}-\mathrm{MSE}$, and the lengths of 95\% CRIs of $\alpha$ under LINEX functions when $c=1, h=1.5, b=0.15234, a=0.6119$, $\alpha=4.36534$, and $\lambda=1$.

\begin{tabular}{lcccccccc}
\hline$n$ & $(r, k)$ & $T$ & $\widehat{\alpha}_{\text {BL }}$ & MSE & Length & $\widehat{\alpha}_{\text {EBL }}$ & $E-$ MSE & Length \\
\hline \multirow{2}{*}{50} & \multirow{2}{*}{$(40,30)$} & 0.2 & 4.03923 & 0.85724 & 4.03967 & 3.85311 & 0.76053 \\
& & 0.4 & 4.09352 & 0.65205 & 3.15369 & 3.93734 & 0.52786 & 2.73469 \\
\multirow{2}{*}{80} & \multirow{2}{*}{$(60,40)$} & 0.2 & 4.11342 & 0.44727 & 2.66239 & 3.97737 & 0.43597 \\
& & 0.4 & 4.20233 & 0.37891 & 2.29636 & 4.07946 & 0.35767 & 2.61368 \\
\multirow{2}{*}{120} & \multirow{2}{*}{$(90,60)$} & 0.2 & 4.17934 & 0.33085 & 2.26593 & 4.08631 & 0.31428 \\
\end{tabular}

TABLE 3: $\widehat{R(t)}_{\mathrm{BS}}, \widehat{R(t)}_{\mathrm{EBS}}$, MSE, E-MSE, and the lengths of 95\% CRIs of $R(t)$ under SEL functions when $c=1, h=1.5, b=0.15234$, $a=0.6119, \alpha=4.36534$, and $\lambda=1, t=0.7$

\begin{tabular}{ccccccccc}
\hline$n$ & $(r, k)$ & $T$ & $\widehat{R(t}_{\mathrm{BS}}$ & MSE & Length & $\widehat{R(t)})_{\mathrm{EBS}}$ & $E-$ MSE & Length \\
\hline \multirow{2}{*}{50} & \multirow{2}{*}{$(40,30)$} & 0.2 & 0.689468 & 0.0495884 & 0.143986 & 0.684285 & 0.0433121 & 0.133462 \\
& \multirow{2}{*}{80} & 0.4 & 0.665547 & 0.0314824 & 0.142313 & 0.654002 & 0.0279769 & 0.132002 \\
\hline \multirow{2}{*}{120} & \multirow{2}{*}{$(60,40)$} & 0.2 & 0.542086 & 0.0202652 & 0.134112 & 0.534464 & 0.0181541 & 0.127219 \\
& \multirow{2}{*}{$(90,60)$} & 0.4 & 0.568990 & 0.0156474 & 0.132062 & 0.564247 & 0.0138038 & 0.126219 \\
\hline
\end{tabular}

TABLE 4: $\widehat{R(t)}_{\mathrm{BL}}, \widehat{R(t)}_{\mathrm{EBL}}, \mathrm{MSE}, \mathrm{E}-\mathrm{MSE}$, and the lengths of $95 \%$ CRIs of $R(t)$ under LINEX functions when $c=1, h=1.5, b=0.15234$, $a=0.6119, \alpha=4.36534, \lambda=1$, and $t=0.7$.

\begin{tabular}{lcccccccc}
\hline$n$ & $(r, k)$ & $T$ & $\widehat{R(t)}_{\mathrm{BL}}$ & MSE & Length & $\widehat{R(t}_{\mathrm{EBL}}$ & $E-$ MSE & Length \\
\hline \multirow{2}{*}{50} & \multirow{2}{*}{$(40,30)$} & 0.2 & 0.683318 & 0.0509062 & 0.144954 & 0.679177 & 0.0451324 & 0.134901 \\
& \multirow{2}{*}{80} & 0.4 & 0.662474 & 0.0326797 & 0.143429 & 0.656167 & 0.0298591 & 0.133946 \\
\hline \multirow{2}{*}{120} & \multirow{2}{*}{$(60,40)$} & 0.2 & 0.556141 & 0.0281050 & 0.135667 & 0.552881 & 0.0244444 & 0.129128 \\
& \multirow{2}{*}{$(90,60)$} & 0.4 & 0.582577 & 0.0168338 & 0.133724 & 0.581692 & 0.0152605 & 0.127041 \\
\hline
\end{tabular}

$$
\begin{aligned}
\operatorname{MSE}(\widehat{\alpha}) & =\frac{1}{10000} \sum_{j=1001}^{10000}\left(\widehat{\alpha}_{j}-\alpha\right)^{2}, \\
\operatorname{MSE}(\widehat{R}(t)) & =\frac{1}{10000} \sum_{j=1001}^{10000}\left(\widehat{R}(t)_{j}-R(t)\right)^{2},
\end{aligned}
$$

in which $\widehat{\alpha}$ and $\widehat{R}(t)$ are the estimations of $\alpha$ and $R(t)$.

Under two types of loss functions, we get the 95\% CRIs for $\alpha$ from (52). For $R(t)$, we get the same kind of CRIs from (53). Then, we calculate the length of the CRIs.

Tables 1-4 give average Bayesian and E-Bayesian estimations, MSE, E-MSE, and the lengths of 95\% CRIs for different values of estimation. Under both SEL and
LINEX loss functions, $\alpha$ is estimated by the method in Section 4. For the shape parameter, Bayesian and EBayesian estimation are got. For the length of CRIs, we use $\mathrm{MH}$ algorithm to generate Markov chain and then select the appropriate quantile and subtract the minimum from the maximum. Similarly, we can calculate the value of the estimation of $R(t)$ by using the samples in Markov chain. Numerical results are computed under the condition when changing the values of $n, r, k$, or changing the time $T$, we investigate their effects on the accuracy of the proposed method when increasing their values.

From Tables 1-4, we can get the following information about the estimated value. The first is the comparison of the effectiveness of E-Bayesian estimation and Bayesian estimation. We observe that the E-MSE of E-Bayesian 
estimations of $\alpha$ is generally smaller comparing to the MSE of Bayesian estimations. So, E-Bayesian estimation of parameters is more effective. For $R(t)$, we can also conclude that the E-Bayesian estimation is more effective by observing the table.

Next, we observe the estimation under two loss functions. We can find that under the SEL function, the E-MSE of Bayesian and E-Bayesian estimations are mostly less than that under the LINEX loss function. For MSE, the same result is obtained. Therefore, through the comparison of MSE, we can conclude that Bayesian estimation is more effective under the SEL function than that under the LINEX loss function. Through the comparison of E-MSE, we can also conclude that E-Bayesian estimation is more effective under the SEL function than that under the LINEX loss function.

On the other hand, for Bayesian estimation, when we increase $n, r$, and $T$, the MSE decreases obviously. For EBayesian estimation, when we increase the same values, the E-MSE also decreases. Furthermore, when $n$ is fixed, the lengths of CRIs for $\alpha$ and $R(t)$ decrease with the increase of $r, k$, and $T$. Based on the above analysis, we get the conclusion that the larger the sample size $n$ is, the better the estimation is, when the E-MSE is smaller than the MSE.

In short, from the condition analysis in this section, compared with Bayesian estimation, E-Bayesian estimation can improve the effectiveness of the estimation. Increasing the sample size, prolonging the specified time, or using SEL function can improve the effectiveness of the two estimates at the same time.

Finally, all validity judgments are based on the comparison between MSE and E-MSE. We can get the conclusion that there is a relationship between $E-\operatorname{MSE}\left(\widehat{\alpha}_{\mathrm{EBS}}\right)$ and $E-\operatorname{MSE}\left(\widehat{\alpha}_{\mathrm{BS}}\right)$ as

$$
E-\operatorname{MSE}\left(\widehat{\alpha}_{\mathrm{EBS}}\right)<\operatorname{MSE}\left(\widehat{\alpha}_{\mathrm{BS}}\right) .
$$

Similarly, when $h=1.5, E-\operatorname{MSE}\left(\widehat{\alpha}_{\mathrm{EBL}}\right)$ and $E-\operatorname{MSE}\left(\widehat{\alpha}_{\mathrm{BL}}\right)$ have the relationship

$$
E-\operatorname{MSE}\left(\widehat{\alpha}_{\mathrm{EBL}}\right)<\operatorname{MSE}\left(\widehat{\alpha}_{\mathrm{BL}}\right) \text {. }
$$

Under SEL and LINEX loss functions, $E-\operatorname{MSE}\left(\widehat{\alpha}_{\mathrm{EBS}}\right)$ and $E-\operatorname{MSE}\left(\widehat{\alpha}_{\mathrm{EBL}}\right)$ meet the relationship

$$
E-\operatorname{MSE}\left(\widehat{\alpha}_{\mathrm{EBS}}\right)<E-\operatorname{MSE}\left(\widehat{\alpha}_{\mathrm{EBL}}\right) \text {. }
$$

\section{Numerical Results}

In this section, we deal with four different cases of samples generated from Lomax distribution by the applying of (54) based on Type-I HCS. We can see the effects when changing the prescribed experimental time, $T$. The conclusions are as follows:

(i) We generate a set of sample data which obey the Lomax distribution and set three integers $n=40, r=$ $30, k=20$ and the time $T=0.6$. The data is given as follows: $0.0167,0.0168,0.0194,0.0529,0.0563$, $0.0870,0.0953,0.1093,0.1438,0.1578,0.1585,0.1620$,
$0.1673,0.1816,0.1983,0.2090,0.2160,0.2161,0.2175$, $0.2209,0.2389,0.2693,0.2816,0.2868,0.3307$, $0.4528,0.4831,0.4992,0.6587,0.7952,0.9217,1.0830$, $1.2384,1.5641,1.5848,1.6500,1.6603,2.0739$, 2.3283, and 3.4375 .

$X_{k: n}=X_{20: 40}=0.2209<T$, the end time of the test, is

$T_{*}=\min \left\{T, X_{r: n}\right\}=\min \{0.6,0.7952\}=0.6$.

We observe that only 28 out of 40 items have failed. The final experimental time is $T=0.6$.

(ii) We generate a set of sample data which obey the Lomax distribution and set three integers $n=40, r=$ $35, k=30$ and time $T=0.8$. The data is given as follows: $0.0029,0.0268,0.0323,0.0345,0.0391$, 0.0468, 0.0470, 0.0496, 0.0920, 0.1021, 0.1121, 0.1261, $0.1321,0.1397,0.1540,0.1585,0.1886,0.2077,0.2083$, $0.2223,0.2265,0.2308,0.2318,0.2417,0.32197$, $0.3689,0.4559,0.4667,0.4734,0.5467,0.5954$, $0.6951,0.8034,0.8408,0.8706,0.9446,1.3118,2.5586$, 2.7567, and 3.1102 .

$$
\begin{aligned}
X_{k: n} & =X_{30: 40}=0.5467<T, \text { the test, is terminated at } \\
T_{*} & =\min \left\{T, X_{r: n}\right\}=\min \{0.8,0.8706\}=0.8 .
\end{aligned}
$$

We observe that only 32 out of 40 items have failed. The final experimental time is $T=0.8$. So we find out, the value of $T$ can not only influence the termination time of the lifetesting experiments but also determine the number of failed items.

6.1. The Fitted Curve of PDF. In Table 5, there is a group of data generated from a type of Lomax distribution. From the dataset, when $\lambda=1, h=0.7$, and $c=1$, we can compute $\widehat{\alpha}_{B}$, the Bayesian estimation of $\alpha$, and its $\operatorname{MSE}\left(\widehat{\alpha}_{B}\right)$. Besides, we compute $\widehat{\alpha}_{\mathrm{EB}}$, the E-Bayesian of $\alpha$, and get its $E-\operatorname{MSE}\left(\widehat{\alpha}_{B}\right)$. We can get the following estimation and mean square error under SEL function as follows:

(i) $\widehat{\alpha}_{B}=2.1836, \operatorname{MSE}\left(\widehat{\alpha}_{B}\right)=0.1403$.

(ii) $\widehat{\alpha}_{\mathrm{EB}}=2.2092, E-\operatorname{MSE}\left(\widehat{\alpha}_{B}\right)=0.1214$.

From these data, we can observe the density of $X$ we gained and the chart of $\operatorname{Lomax}\left(\widehat{\alpha}_{B}, 1\right)$ and $\operatorname{Lomax}\left(\widehat{\alpha}_{\mathrm{EB}}, 1\right)$ by putting them in the same box, as shown in Figures 5 and 6 . In Figure 6, E-Estimated means the expectation of the estimation.

To analyze these datasets quantitatively, the Kolmogorov-Smirnov (K-S) test is applied under the cumulative distribution function. With this kind of test, we are able to study whether two empirical distributions are close to each other, or whether one empirical distribution is close to another ideal distribution. We deal with our distributions and datasets in two steps. First, we generate the cumulative probability graph of two distributions. Then, we measure the distance of the given $x$ value between two curves along the $y$ axis. Here, we need to introduce a vector $\theta=(A, p)$. For each $x$ value, we compute the distance and compare it with 
TABle 5: Dataset 1.

\begin{tabular}{|c|c|c|c|c|c|c|c|c|c|}
\hline 0.0169 & 0.1543 & 0.2641 & 0.4035 & 1.0320 & 0.0201 & 0.1598 & 0.2901 & 0.4339 & 1.0698 \\
\hline 0.0295 & 0.1640 & 0.2911 & 0.4555 & 1.3370 & 0.0521 & 0.1766 & 0.2945 & 0.5886 & 1.3473 \\
\hline 0.0565 & 0.1853 & 0.2990 & 0.5922 & 1.4437 & 0.0810 & 0.2015 & 0.2997 & 0.6229 & 1.7470 \\
\hline 0.0979 & 0.2146 & 0.3254 & 0.6335 & 1.9646 & 0.1020 & 0.2199 & 0.3728 & 0.6475 & 4.2160 \\
\hline 0.1133 & 0.2228 & 0.3870 & 0.6748 & 6.0603 & 0.1350 & 0.2342 & 0.4007 & 0.6992 & 9.7196 \\
\hline
\end{tabular}

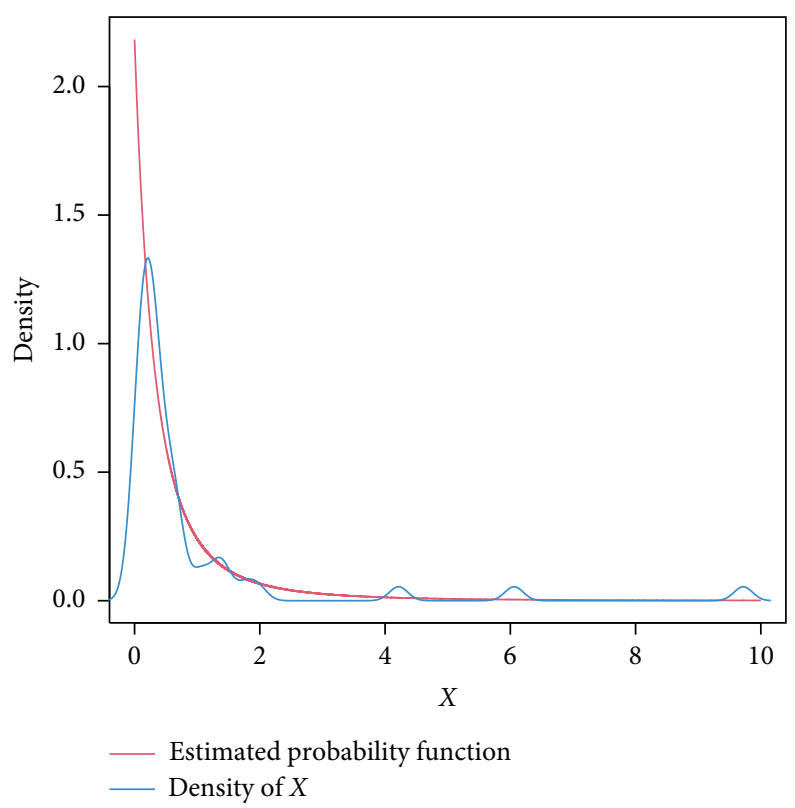

FIgURE 5: The fitted curve of estimated PDF.

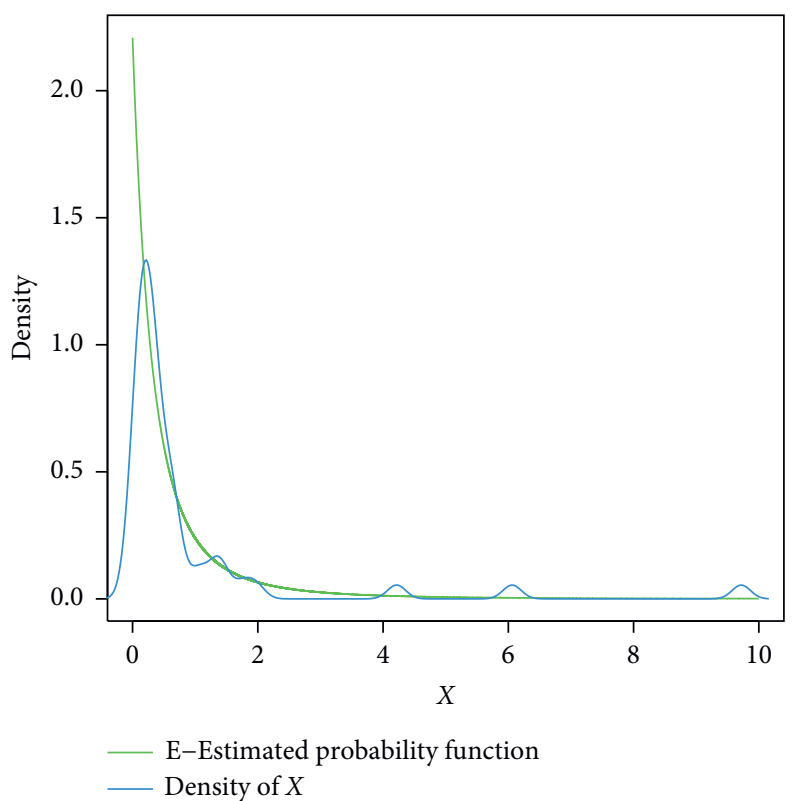

FIgURE 6: The fitted curve of E-Estimated PDF.

others. We pick out the maximum distance $A$. After that, we insert the maximum distance into the K-S probability function to calculate the probability value $p$ ( $p$ value). The lower the probability value is, the more likely the two distributions are different. On the contrary, the higher or closer to 1 the value is, the more similar the two distributions are. In general, if $p>0.05$, we can suppose that the data can be analyzed by the function we studied. For more details about $\mathrm{K}-\mathrm{S}$ test, we can refer to Lopes [20].

We define the null hypothesis as follows: 
(i) $H_{0}$ : Lomax distribution can be used as the distribution function of data fitting.

According to Figures 3 and 4, both the Bayesian estimation probability density function and E-Bayesian estimation probability density function are close to the distribution of $X$. We put the K-S distance, $A$, and the associated $p$ value, $p$, into a vector $\theta=(A, p)$.

We get that $\theta=(0.1514,0.2042)$. So, for Table 5 , we cannot reject the $H_{0}$. So, it is appropriate to use this method to learn other estimations under different loss functions.

6.2. Data Generated from Lomax Distribution. There is another group of data generated from Lomax distribution in Table 6.

We use the method described in the previous section and the dataset above to perform the Bayesian estimation of $\alpha$ and $R(t)$. After that, we can get their E-Bayesian estimation. Use $\mathrm{MH}$ algorithm, when $h=1.5, \lambda=1, c=0.1, t=0.07$ and $T=0.2, k=30, r=40, n=50$; we are able to get the following estimation and mean square error under two loss functions, SEL and LINEX, as follows:

(i) $\widehat{\alpha}_{\mathrm{BS}}=8.6394, \operatorname{MSE}\left(\widehat{\alpha}_{\mathrm{BS}}\right)=0.7218$.

(ii) $\widehat{\alpha}_{\mathrm{EBS}}=8.6463, E-\operatorname{MSE}\left(\widehat{\alpha}_{\mathrm{EBS}}\right)=0.6939$.

(iii) $\widehat{\alpha}_{\mathrm{BL}}=8.6161, \operatorname{MSE}\left(\widehat{\alpha}_{\mathrm{BL}}\right)=0.7166$.

(iv) $\widehat{\alpha}_{\mathrm{EBL}}=8.6230, E-\operatorname{MSE}\left(\widehat{\alpha}_{\mathrm{EBL}}\right)=0.6843$.

(v) $\widehat{R}(t)_{\mathrm{BS}}=0.59192, \operatorname{MSE}\left(\widehat{R}(t)_{\mathrm{BS}}\right)=0.01218$.

(vi) $\widehat{R}(t)_{\mathrm{EBS}}=0.59188, E-\operatorname{MSE}\left(\widehat{R}(t)_{\mathrm{EBS}}\right)=0.01139$.

(vii) $\widehat{R}(t)_{\mathrm{BL}}=0.59173, \operatorname{MSE}\left(\widehat{R}(t)_{\mathrm{BL}}\right)=0.01137$.

(viii) $\widehat{R}(t)_{\mathrm{EBL}}=0.59169, E-\operatorname{MSE}\left(\widehat{R}(t)_{\mathrm{EBL}}\right)=0.01043$.

The line chart of the density of both $\alpha_{B}$ and $\alpha_{\mathrm{EB}}$ is presented in Figure 7. Through the comparison of the above results, we get the conclusion that

(i) $\widehat{\alpha}_{\mathrm{BS}}<\widehat{\alpha}_{\mathrm{EBS}}, \operatorname{MSE}\left(\widehat{\alpha}_{\mathrm{BS}}\right)>E-\operatorname{MSE}\left(\widehat{\alpha}_{\mathrm{EBS}}\right)$,

(ii) $\widehat{\alpha}_{\mathrm{BL}}<\widehat{\alpha}_{\mathrm{EBL}}, \operatorname{MSE}\left(\widehat{\alpha}_{\mathrm{BL}}\right)>E-\operatorname{MSE}\left(\widehat{\alpha}_{\mathrm{EBL}}\right)$.

From the analysis of Table 6 , we can get the conclusion that under two loss functions, LINEX and SEL functions, $E-\operatorname{MSE}\left(\widehat{\alpha}_{\mathrm{EB}}\right)$ is less than $\operatorname{MSE}\left(\widehat{\alpha}_{B}\right)$ and $E-\operatorname{MSE}\left(\widehat{R}(t)_{\mathrm{EB}}\right)$ is less than $\operatorname{MSE}\left(\widehat{R}(t)_{\mathrm{B}}\right)$. In application aspect, E-Bayesian estimation is more effective than Bayesian estimation.

\section{Real Data Analysis}

For the purpose of illustration, three real datasets are analyzed in this section. The applicability of the E-Bayesian estimation for Lomax distribution is presented by analyzing the numerical examples. First, we will prove that it is feasible to analyze the data with Lomax distribution. Next, we will process the data under Type-I HCS.

7.1. An Illustrative Example. Numerical examples verify the applicability of the above methods. We can find the feasible data in the experimental data of physical research, and we can use the Lomax distribution to fit the experimental data that obey the life distribution. As long as the data can pass K$\mathrm{S}$ test, we can use Lomax distribution for data analysis. In fact, the same method can be used for other life distributions too. As long as the fitting degree of the life distribution function falls in the specified range, it can be used for research. Now, we use the method of the previous study to analyze two sets of data. Bennett and Filliben [21] provided these datasets. These datasets represent the minority electron mobility for P-type $G a_{1-x} A l_{x} A s$, a kind of salt compound, with seven different values of mole fraction $\left(x_{B}\right)$. More information about data sources can be obtained from Bennett and Filliben [21]. We choose two $x_{B}$ and use the real datasets related to them in our study. Both real datasets containing 21 observations are shown in Tables 7 and 8 .

Kolmogorov-Smirnov (K-S) tests are conducted to test whether the datasets can be analyzed by the Lomax distribution.

We define the null hypothesis as follows:

(i) $H_{01}$ : Lomax distribution can fit real dataset 1 .

(ii) $H_{02}$ : Lomax distribution can fit real dataset 2.

Since $\theta_{1}=(0.2857,0.365)$ and $\theta_{2}=(0.3333,0.1963)$, so we cannot reject the $H_{01}$ and $H_{02}$.

We assume that the values of real datasets 1 and 2 are failure life-testing experiment observations following the Lomax distribution. By applying generalized Type-I HCS on these uncensored data, considering $\lambda=3.8, n=21, r=15$, $k=10, T=2, a=0.6119, b=0.15, h=1.5, c=1$, and $t=$ 0.7 , we analyze two datasets and obtain the experimental time and different estimations under two loss functions, SEL and LINEX.

For real dataset 1 ,

(i) $X_{k: n}=X_{10: 21}=1.296<T$. The end time of the experiment is

$T_{*}=\min \left\{X_{r: n}, T\right\}=\min \{1.715,2\}=1.715$.

Only 15 out of 21 items have failed. The final experimental time is $T=1.715$.

(ii) $\widehat{\alpha}_{\mathrm{BS}}=2.41501, \widehat{\alpha}_{\mathrm{EBS}}=2.27865$.

(iii) $\widehat{\alpha}_{\mathrm{BL}}=2.17180, \widehat{\alpha}_{\mathrm{EBL}}=2.05884$.

(iv) $\widehat{R}(t)_{\mathrm{BS}}=0.66477, \widehat{R}(t)_{\mathrm{EBS}}=0.68027$.

(v) $\widehat{R}(t)_{\mathrm{BL}}=0.69267, \widehat{R}(t)_{\mathrm{EBL}}=0.70603$.

For the MSE and E-MSE of the estimations of $\alpha$,

(i) $\operatorname{MSE}\left(\widehat{\alpha}_{\mathrm{BS}}\right)=0.37358$,

(ii) $\operatorname{MSE}\left(\widehat{\alpha}_{\mathrm{BL}}\right)=0.43273$,

(iii) $E-\operatorname{MSE}\left(\widehat{\alpha}_{\mathrm{EBS}}\right)=0.33559$,

(iv) $E-\operatorname{MSE}\left(\widehat{\alpha}_{\mathrm{EBL}}\right)=0.38422$.

For Real dataset 2,

(i) $X_{k: n}=X_{10: 21}=1.165<T$. The end time of the experiment is

$$
T_{*}=\min \left\{X_{r: n}, T\right\}=\min \{1.53,2\}=1.53 .
$$


TABLe 6: Dataset 2.

\begin{tabular}{llllllllll}
\hline 0.0018 & 0.0037 & 0.0071 & 0.0096 & 0.0010 & 0.0139 & 0.0147 & 0.0193 & 0.0198 & 0.0202 \\
0.0234 & 0.0293 & 0.0295 & 0.0312 & 0.0314 & 0.0328 & 0.0392 & 0.0464 & 0.0483 & 0.0491 \\
0.0507 & 0.0516 & 0.0532 & 0.0543 & 0.0560 & 0.0611 & 0.0701 & 0.0705 & 0.0726 & 0.0804 \\
0.0806 & 0.0845 & 0.0881 & 0.0890 & 0.0908 & 0.0923 & 0.1020 & 0.1097 & 0.1254 & 0.1337 \\
0.1341 & 0.1546 & 0.1635 & 0.1992 & 0.2132 & 0.2154 & 0.2529 & 0.3969 & 0.5548 & 0.7497 \\
\hline
\end{tabular}

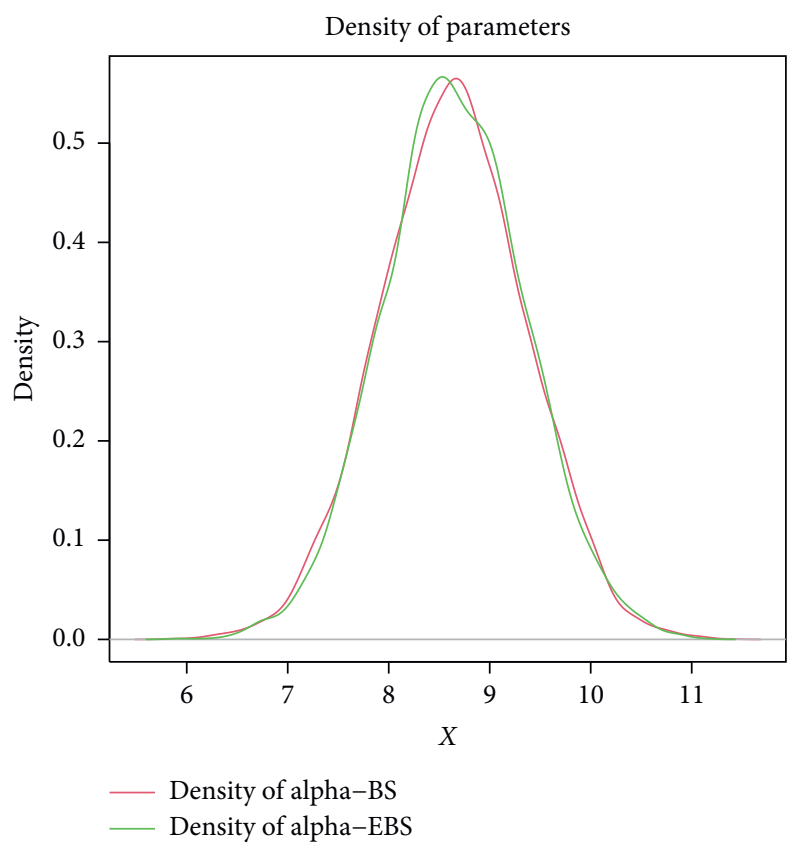

Figure 7: Density of $\alpha$ and $\alpha_{E}$.

TABle 7: Real dataset $1\left(x_{B}=0.25\right)$.

\begin{tabular}{lcccccc}
\hline 0.700 & 1.296 & 1.154 & 1.016 & 1.525 & 0.6292 & 0.7948 \\
1.715 & 2.779 & 3.051 & 2.371 & 2.214 & 2.045 & 0.604 \\
1.528 & 1.506 & 0.8881 & 1.115 & 1.397 & 0.6449 & 0.6175 \\
\hline
\end{tabular}

TABLe 8: Real dataset $2\left(x_{B}=0.30\right)$.

\begin{tabular}{|c|c|c|c|c|c|c|}
\hline 1.368 & 1.002 & 0.8013 & 0.5873 & 1.250 & 1.347 & 0.5647 \\
\hline 0.7241 & 1.165 & 0.576 & 0.9198 & 1.366 & 0.6403 & 1.041 \\
\hline 1.959 & 1.814 & 2.288 & 2.092 & 2.658 & 2.434 & 1.530 \\
\hline
\end{tabular}

Only 15 out of 21 items have failed. The final experimental time is $T=1.53$.

(ii) $\widehat{\alpha}_{\mathrm{BS}}=2.89033, \widehat{\alpha}_{\mathrm{EBS}}=2.72143$.

(iii) $\widehat{\alpha}_{\mathrm{BL}}=2.58394, \widehat{\alpha}_{\mathrm{EBL}}=2.44568$.

(iv) $\widehat{R}(t)_{\mathrm{BS}}=0.61343, \widehat{R}(t)_{\mathrm{EBS}}=0.63120$.

(v) $\widehat{R}(t)_{\mathrm{BL}}=0.64605, \widehat{R}(t)_{\mathrm{EBL}}=0.66133$.

For the MSE and E-MSE of the estimations of $\alpha$,

(i) $\operatorname{MSE}\left(\widehat{\alpha}_{\mathrm{BS}}\right)=0.47434$,

(ii) $\operatorname{MSE}\left(\widehat{\alpha}_{\mathrm{BL}}\right)=0.56821$,

(iii) $E-\operatorname{MSE}\left(\widehat{\alpha}_{\mathrm{EBS}}\right)=0.42407$,

(iv) $E-\operatorname{MSE}\left(\widehat{\alpha}_{\mathrm{EBL}}\right)=0.50066$.

We can further analyze the characteristics of the datasets; take real dataset 1 as an example. From Table 9, when $\lambda=3.8, T=2, n=21, k=15, r=18, a=0.6119$, and $b=$ 0.15 are fixed in advance, we find that for a value of $c$, $(c=0.25,0.50,0.75,1.00,1.25)$; the values of $\widehat{\alpha}_{\mathrm{EBS}}$ and $\widehat{\alpha}_{\mathrm{EBL}}$ are close to each other. The values of $E-\operatorname{MSE}\left(\widehat{\alpha}_{\mathrm{EBS}}\right)$ and $E-\operatorname{MSE}\left(\widehat{\alpha}_{\mathrm{EBL}}\right)$, when $h=1.5$, have the relationship as follows:

$$
E-\operatorname{MSE}\left(\widehat{\alpha}_{\mathrm{EBS}}\right)<E-\operatorname{MSE}\left(\widehat{\alpha}_{\mathrm{EBL}}\right)
$$

Moreover, the values of $\widehat{\alpha}_{\mathrm{EBS}}, \widehat{\alpha}_{\mathrm{EBL}}$ and $E-\operatorname{MSE}\left(\widehat{\alpha}_{\mathrm{EBS}}\right)$, $E-\operatorname{MSE}\left(\widehat{\alpha}_{\mathrm{EBL}}\right)$ all have a clear law of changing for the different values of $c,(c=0.25,0.50,0.75,1.00,1.25)$. We can get the changing trend of $\widehat{\alpha}_{\mathrm{EBS}}, \widehat{\alpha}_{\mathrm{EBL}}$ and $E-\operatorname{MSE}\left(\widehat{\alpha}_{\mathrm{EBS}}\right), E-$ $\operatorname{MSE}\left(\widehat{\alpha}_{\mathrm{EBL}}\right)$ from Figures 8 and 9 .

For $E-\operatorname{MSE}\left(\widehat{\alpha}_{\mathrm{EBS}}\right)$ and $E-\operatorname{MSE}\left(\widehat{\alpha}_{\mathrm{BS}}\right)$, their values have the relationship as follows: 
TABLE 9: Results of $\widehat{\alpha}_{\mathrm{EBS}}, E-\operatorname{MSE}\left(\widehat{\alpha}_{\mathrm{EBS}}\right), \widehat{\alpha}_{\mathrm{EBL}}$, and $E-\operatorname{MSE}\left(\widehat{\alpha}_{\mathrm{EBL}}\right)$ for real dataset 1.

\begin{tabular}{lcccc}
\hline$c$ & $\widehat{\alpha}_{\text {EBS }}$ & $E-\operatorname{MSE}\left(\widehat{\alpha}_{\text {EBS }}\right)$ & $\widehat{\alpha}_{\text {EBL }}$ & $E-$ MSE $\left(\widehat{\alpha}_{\text {EBL }}\right)$ \\
\hline 0.25 & 2.4073 & 0.37393 & 2.1640 & 0.43317 \\
0.50 & 2.3623 & 0.36021 & 2.1274 & 0.41551 \\
0.75 & 2.3195 & 0.34746 & 2.0924 & 0.39925 \\
1.00 & 2.2786 & 0.33559 & 2.0588 & 0.38422 \\
1.25 & 2.2397 & 0.32450 & 2.0267 & 0.37029 \\
\hline
\end{tabular}

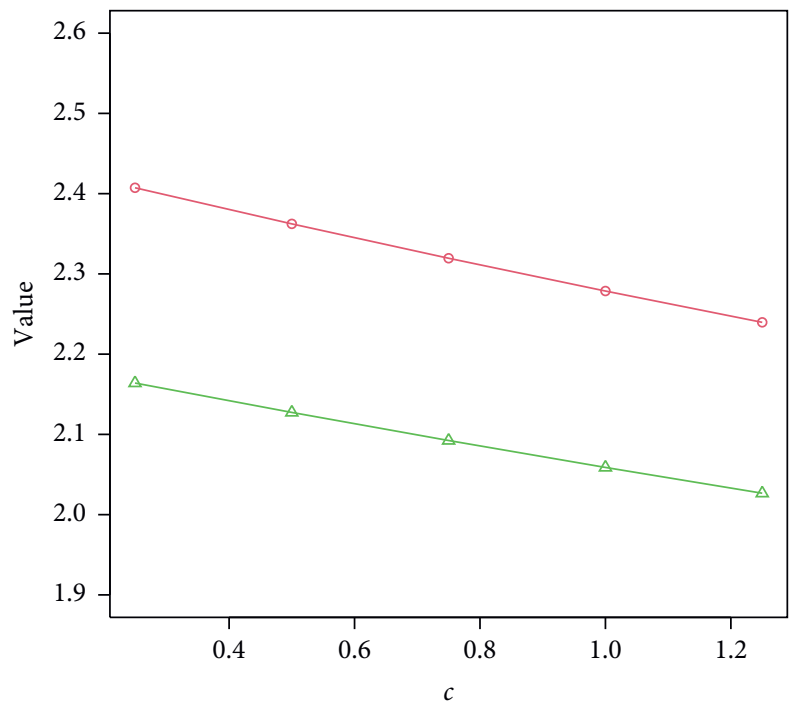

- AlphahatBS

- AlphahatBL

FIgURE 8: Relationship between $c$ and $\widehat{\alpha}_{\mathrm{EBS}}, \widehat{\alpha}_{\mathrm{EBL}}$.

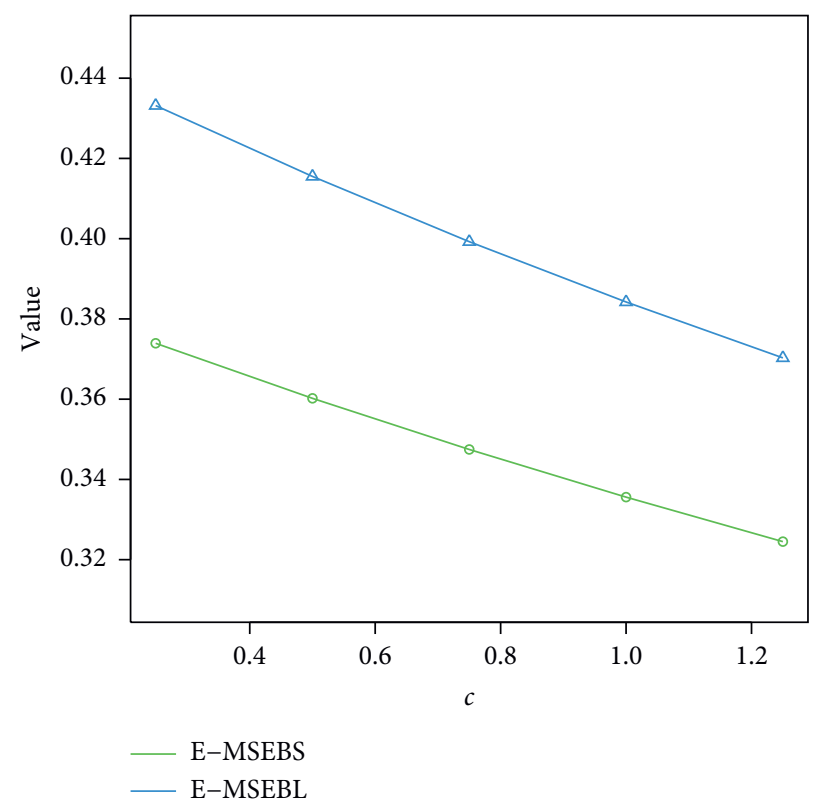

Figure 9: Relationship between $c$ and $E-\operatorname{MSE}\left(\widehat{\alpha}_{\mathrm{EBS}}\right), E-\operatorname{MSE}\left(\widehat{\alpha}_{\mathrm{EBL}}\right)$. 
TABLE 10: Lifetime of ruby laser at energy level $2 E\left(\times 10^{-3} \mathrm{~s}\right)$.

\begin{tabular}{lllll}
\hline 0.0463 & 0.1423 & 0.5417 & 0.5699 & 0.7799 \\
0.8082 & 0.8711 & 1.2083 & 1.2743 & \\
1.6067 & 1.8969 & 2.0418 & 2.5928 & 2.9511 \\
3.8206 & 4.0736 & 4.1606 & 5.1832 & 5.4803 \\
6.4376 & 6.7442 & 7.6308 & 8.1012 & 11.006 \\
\hline
\end{tabular}

TABLE 11: Results of $\widehat{\alpha}_{\mathrm{EBS}}, E-\operatorname{MSE}\left(\widehat{\alpha}_{\mathrm{EBS}}\right), \widehat{\alpha}_{\mathrm{EBL}}$, and $E-\operatorname{MSE}\left(\widehat{\alpha}_{\mathrm{EBL}}\right)$ for lifetime of ruby laser at energy level $2 E\left(\times 10^{-3} \mathrm{~s}\right)$.

\begin{tabular}{lcccc}
\hline$c$ & $\widehat{\alpha}_{\text {EBS }}$ & $E-\operatorname{MSE}\left(\widehat{\alpha}_{\text {EBS }}\right)$ & $\widehat{\alpha}_{\text {EBL }}$ & $E-\operatorname{MSE}\left(\widehat{\alpha}_{\text {EBL }}\right)$ \\
\hline 0.25 & 2.2354 & 0.24377 & 2.0703 & 0.27102 \\
0.50 & 2.2057 & 0.23738 & 2.0448 & 0.26331 \\
0.75 & 2.1771 & 0.23132 & 2.0201 & 0.25603 \\
1.00 & 2.1494 & 0.22557 & 1.9961 & 0.24914 \\
1.25 & 2.1226 & 0.22009 & 1.9729 & 0.24262 \\
\hline
\end{tabular}

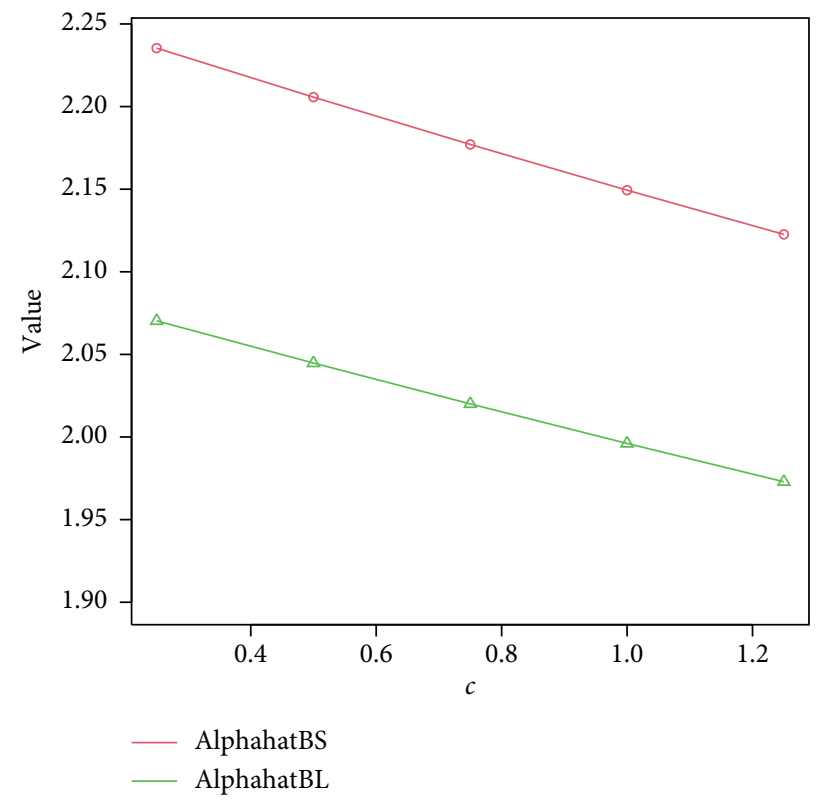

FIGURE 10: Relationship between $c$ and $\widehat{\alpha}_{\mathrm{EBS}}, \widehat{\alpha}_{\mathrm{EBL}}$.

$$
E-\operatorname{MSE}\left(\widehat{\alpha}_{\mathrm{EBS}}\right)<\operatorname{MSE}\left(\widehat{\alpha}_{\mathrm{BS}}\right)
$$

For $E-\operatorname{MSE}\left(\widehat{\alpha}_{\mathrm{EBL}}\right)$ and $E-\operatorname{MSE}\left(\widehat{\alpha}_{\mathrm{BL}}\right)$, their values have the relationship as follows:

$$
E-\operatorname{MSE}\left(\widehat{\alpha}_{\mathrm{EBL}}\right)<\operatorname{MSE}\left(\widehat{\alpha}_{\mathrm{BL}}\right),
$$

when $h=1.5, c=1$.

7.2. A Hypothesis of Laser Energy Level Lifetime Distribution. High-energy atoms emit spontaneous radiation and a photon with energy $h v$. This process is called spontaneous transition. After the spontaneous transition occurs at time $t$, when the ratio of the number of high-level atoms to the number of atoms at the beginning is equal to $1 / e$, the time $t$ at which the energy is reduced to this level is defined as the energy level lifetime.

From the principle of laser, we can know that the average lifetime of the ruby laser at an energy level $2 E$ is about $3 \times 10^{-3} \mathrm{~s}$. Suppose we do not know the lifetime distribution of the laser at the energy level. Relying on a set of experimental data we get from the laboratory, we can guess that it obeys the Lomax distribution. We apply the K-S test as follows:

(i) $H_{0}$ : Lomax distribution can be used to fit the data in Table 10 .

Because $\theta=(0.12,0.8693)$, we cannot reject $H_{0}$.

Use generalized Type-I HCS on these uncensored data, when $h=1.5, \lambda=5.8, c=1, t=0.7$ and $T=6, k=15$, $r=20, n=25$; we are able to compute the estimations of the parameters under two loss functions, SEL and LINEX, as follows:

(i) $\widehat{\alpha}_{\mathrm{BS}}=2.24132, \widehat{\alpha}_{\mathrm{EBS}}=2.14939$.

(ii) $\widehat{\alpha}_{\mathrm{BL}}=2.07626, \widehat{\alpha}_{\mathrm{EBL}}=1.99612$.

At the same time, we can conclude that

(i) $\widehat{R}(t)_{\mathrm{BS}}=0.77462, \widehat{R}(t)_{\mathrm{EBS}}=0.78277$. 


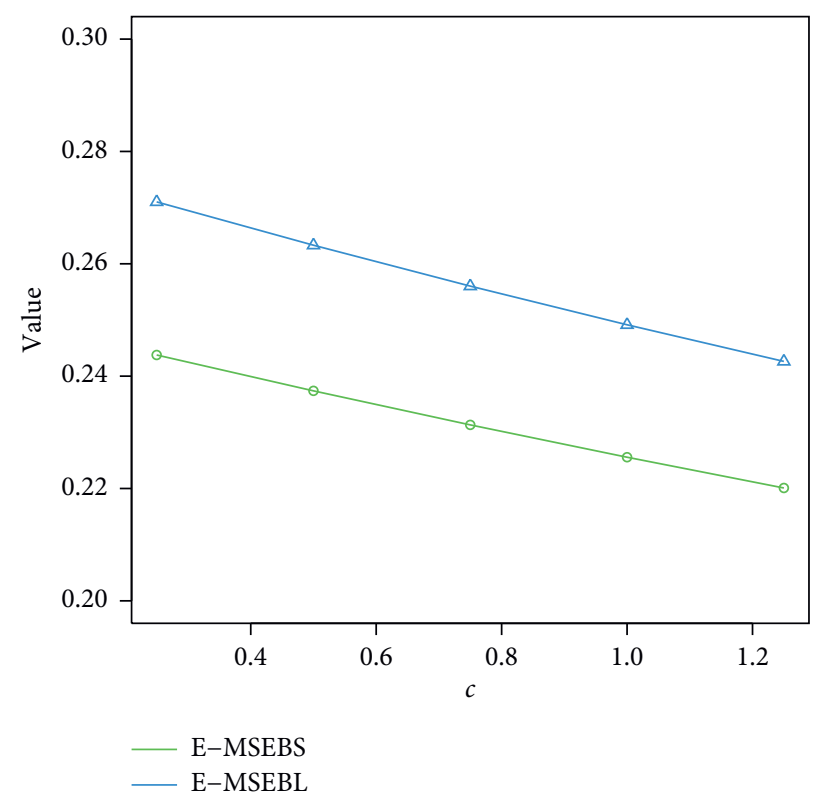

FIgUre 11: Relationship between $c$ and $E-\operatorname{MSE}\left(\widehat{\alpha}_{\mathrm{EBS}}\right), E-\operatorname{MSE}\left(\widehat{\alpha}_{\mathrm{EBL}}\right)$.

(ii) $\widehat{R}(t)_{\mathrm{BL}}=0.78932, \widehat{R}(t)_{\mathrm{EBL}}=0.79657$.

For the MSE and E-MSE of the estimations of $\alpha$,

(i) $\operatorname{MSE}\left(\widehat{\alpha}_{\mathrm{BS}}\right)=0.24372$,

(ii) $\operatorname{MSE}\left(\widehat{\alpha}_{\mathrm{BL}}\right)=0.27097$,

(iii) $E-\operatorname{MSE}\left(\widehat{\alpha}_{\mathrm{EBS}}\right)=0.22557$,

(iv) $E-\operatorname{MSE}\left(\widehat{\alpha}_{\mathrm{EBL}}\right)=0.24914$.

From Table 11, we find that for a value of $c$, ( $c=0.25,0.50,0.75,1.00,1.25)$; the values of $\widehat{\alpha}_{\mathrm{EBS}}$ and $\widehat{\alpha}_{\mathrm{EBL}}$ are close to each other. The values of $E-\operatorname{MSE}\left(\widehat{\alpha}_{\mathrm{EBS}}\right)$ and $E-\operatorname{MSE}\left(\widehat{\alpha}_{\mathrm{EBL}}\right)$, when $h=1.5$, have the relationship as follows:

$$
E-\operatorname{MSE}\left(\widehat{\alpha}_{\mathrm{EBS}}\right)<E-\operatorname{MSE}\left(\widehat{\alpha}_{\mathrm{EBL}}\right) .
$$

Moreover, the values of $\widehat{\alpha}_{\mathrm{EBS}}, \widehat{\alpha}_{\mathrm{EBL}}$ and $E-\operatorname{MSE}\left(\widehat{\alpha}_{\mathrm{EBS}}\right)$, $E-\operatorname{MSE}\left(\widehat{\alpha}_{\mathrm{EBL}}\right)$ all have a clear law of changing for the different values of $c,(c=0.25,0.50,0.75,1.00,1.25)$. We can get the changing trend of $\widehat{\alpha}_{\mathrm{EBS}}, \widehat{\alpha}_{\mathrm{EBL}}$ and $E-\operatorname{MSE}\left(\widehat{\alpha}_{\mathrm{EBS}}\right), E-$ $\operatorname{MSE}\left(\widehat{\alpha}_{\mathrm{EBL}}\right)$ from Figures 10 and 11.

For $E-\operatorname{MSE}\left(\widehat{\alpha}_{\mathrm{EBS}}\right)$ and $E-\operatorname{MSE}\left(\widehat{\alpha}_{\mathrm{BS}}\right)$, their values have the relationship as follows:

$$
E-\operatorname{MSE}\left(\widehat{\alpha}_{\mathrm{EBS}}\right)<\operatorname{MSE}\left(\widehat{\alpha}_{\mathrm{BS}}\right) \text {. }
$$

For $E-\operatorname{MSE}\left(\widehat{\alpha}_{\mathrm{EBL}}\right)$ and $E-\operatorname{MSE}\left(\widehat{\alpha}_{\mathrm{BL}}\right)$, their values have the relationship as follows:

$$
E-\operatorname{MSE}\left(\widehat{\alpha}_{\mathrm{EBL}}\right)<\operatorname{MSE}\left(\widehat{\alpha}_{\mathrm{BL}}\right)
$$

when $h=1.5$ and $c=1$.

For $E-\operatorname{MSE}\left(\widehat{\alpha}_{\mathrm{EBS}}\right)<\operatorname{MSE}\left(\widehat{\alpha}_{\mathrm{BS}}\right), E-\operatorname{MSE}\left(\widehat{\alpha}_{\mathrm{EBL}}\right)<\operatorname{MSE}$ $\left(\widehat{\alpha}_{\mathrm{BL}}\right)$, it is obvious that the E-Bayesian method is more effective in dealing with the data.

\section{Conclusion}

This paper introduces the concept of generalized Type-I hybrid censoring. The E-Bayesian estimation of the unknown parameter is studied based on it. Under two kinds of loss functions, SEL and LINEX, we get the E-Bayesian estimation. By calculating the expected value of MSE, we can get the E-MSE of E-Bayesian estimation. E-MSE plays a key role in the analysis. The E-Bayesian estimation and MSE of $\alpha$ are computed directly by calculus.

The MCMC method is used to analyze the numerical value which is difficult to calculate directly. With the use of the Metropolis-Hastings algorithm, a Markov chain of the parameter $\alpha$ is generated. Using the data in the Markov chain, we can calculate the Bayesian estimation and EBayesian estimation of $R(t)$. At the same time, we are able to obtain the CRIs. Under two loss functions, Bayesian and EBayesian estimations of $\alpha$ and $R(t)$ are computed by the MCMC method. The estimated values are obtained with different sample size $n$. As $n$ changes, we also replace censoring sample sizes $r$ and $k$. When $n$ is a fixed value, different censoring times $T$ are presented. Under different $T, k, r$, and $n$, we get different E-Bayesian estimation results. Besides, three real datasets are provided for illustration. Kolmogorov-Smirnov tests are conducted to prove that the Lomax distribution can be used to analyze the datasets.

The values of $E-\operatorname{MSE}\left(\widehat{\alpha}_{\mathrm{EBS}}\right)$ and $E-\operatorname{MSE}\left(\widehat{\alpha}_{\mathrm{BS}}\right)$ have the following relationship:

$$
E-\operatorname{MSE}\left(\widehat{\alpha}_{\mathrm{EBS}}\right)<\operatorname{MSE}\left(\widehat{\alpha}_{\mathrm{BS}}\right),
$$

and the values of $E-\operatorname{MSE}\left(\widehat{\alpha}_{\mathrm{EBL}}\right)$ and $E-\operatorname{MSE}\left(\widehat{\alpha}_{\mathrm{BL}}\right)$ also have the following relationship:

$$
E-\operatorname{MSE}\left(\widehat{\alpha}_{\mathrm{EBL}}\right)<\operatorname{MSE}\left(\widehat{\alpha}_{\mathrm{BL}}\right),
$$


when $h$ has a definite value.

This article quantitatively shows the result: compared to the Bayesian estimation method, the E-Bayesian estimation method is more efficient.

\section{Data Availability}

The data used to support the findings of this study are available within the article.

\section{Conflicts of Interest}

The authors declare that they have no conflicts of interest.

\section{Acknowledgments}

The authors gratefully acknowledge the financial support provided by the Project 202110004007 which was supported by the National Training Program of Innovation and Entrepreneurship for Undergraduates.

\section{References}

[1] A. Rabie and J. Li, "E-bayesian estimation for burr-X distribution based on generalized type-I hybrid censoring scheme," American Journal of Mathematical and Management Sciences, vol. 39, no. 1, pp. 41-55, 2020.

[2] Z. F. Jaheen and H. M. Okasha, "E-bayesian estimation for the Burr type XII model based on type-2 censoring," Applied Mathematical Modelling, vol. 35, no. 10, pp. 4730-4737, 2011.

[3] S. Liu and W. Gui, "Estimating the parameters of the twoparameter Rayleigh distribution based on adaptive type II progressive hybrid censored data with competing risks," Mathematics, vol. 8, no. 10, p. 1783, 2020.

[4] M. Han, "E-Bayesian estimation and its E-MSE under the scaled squared error loss function, for exponential distribution as example," Communications in Statistics-Simulation and Computation, vol. 48, no. 6, pp. 1880-1890, 2019.

[5] M. Han, "E-Bayesian estimations of parameter and its evaluation standard: E-MSE (expected mean square error) under different loss functions," Communications in Statistics-Simulation and Computation, pp. 1-18, 2019.

[6] M. Han, "The E-Bayesian estimation and its E-MSE of Pareto distribution parameter under different loss functions," Journal of Statistical Computation and Simulation, vol. 90, no. 10, pp. 1834-1848, 2020.

[7] B. Chandrasekar, A. Childs, and N. Balakrishnan, "Exact likelihood inference for the exponential distribution under generalized Type-I and Type-II hybrid censoring," Naval Research Logistics, vol. 51, no. 7, pp. 994-1004, 2004.

[8] M. Al-Sobhi and B. Al-Zahrani, "On parameters estimation of Lomax distribution under general progressive censoring," Journal of Quality and Reliability Engineering, vol. 2013, no. 5, p. 7, 2013.

[9] J. A. Labban, "On 2-parameter estimation of Lomax distribution," Journal of Physics Conference Series, vol. 1294, p. 032018, 2019.

[10] B. Alzahrani and H. Sagor, "The Poisson-Lomax distribution," Revista Colombiana De Estadistica, vol. 37, no. 1, pp. 223-243, 2014.

[11] G. M. Cordeiro, E. M. M. Ortega, and B. V. Popovic, "The Gamma-Lomax distribution," Journal of Statistical Computation and Simulation, vol. 85, no. 1-3, pp. 305-319, 2015.
[12] E. M. Almetwally and H. M. Almongy, "Parameter estimation and stress-strength model of Power Lomax distribution: classical methods and Bayesian estimation," Journal of Data Science, vol. 18, no. 4, pp. 718-738, 2020.

[13] S. K. Ashour and A. M. Abdelfattah, "Parameter estimation of the hybrid censored Lomax distribution," Pakistan Journal of Statistics and Operation Research, vol. 7, no. 1, pp. 1-19, 2010.

[14] S. Wei, C. Wang, and Z. Li, "Bayes estimation of Lomax distribution parameter in the composite LINEX loss of symmetry," Applied Mathematics A Journal of Chinese Universities, vol. 20 , no. 5 , pp. 1277-1287, 2017.

[15] M. Han, "E-bayesian estimation of the exponentiated distribution family parameter under linex loss function," Communications in Statistics, vol. 48, no. 3, pp. 648-659, 2019.

[16] M. Han, "The structure of hierarchical prior distribution and its applications," Chinese Operation Research and Management Science, vol. 6, pp. 31-40, 1997.

[17] M. Han, "E-bayesian estimation of failure probability and its application," Mathematical and Computer Modelling, vol. 45, no. 9-10, pp. 1272-1279, 2007.

[18] W. K. Hastings, "Monte Carlo sampling methods using Markov chains and their application," Biometrika, vol. 57, no. 1, pp. 97-109, 1970.

[19] M. K. Cowles and B. P. Carlin, "Markov chain Monte Carlo diagnostics: a comparative review," Journal of American Statistical Association, vol. 91, no. 434, pp. 883-904, 1995.

[20] R. H. C. Lopes, “Kolmogorov-smirnov test', International Encyclopedia of Statistical Science, vol. 10, no. 1, pp. 718-720, 2014.

[21] H. S. Bennett and J. J. Filliben, “A systematic approach for multidimensional, closed-form analytic modeling: minority electron mobilities in $G a_{1-x} A l_{x}$ As heterostructures," Journal of Research of the National Institute of Standards and Technology, vol. 1, no. 3, pp. 441-452, 2000. 